\title{
Cultes et pratiques rituelles chtoniens
}

à Incoronata

La campagne de 2018

\section{Mario Denti}

\section{(2) OpenEdition Journals}

Édition électronique

URL : http://journals.openedition.org/cefr/3274

DOI : $10.4000 /$ cefr.3274

ISSN : 2282-5703

Éditeur

École française de Rome

Référence électronique

Mario Denti, "Cultes et pratiques rituelles chtoniens à Incoronata », Chronique des activités

archéologiques de l'École française de Rome [En ligne], Italie du Sud, mis en ligne le 30 août 2019,

consulté le 01 septembre 2019. URL : http://journals.openedition.org/cefr/3274; DOI : 10.4000/ cefr.3274

Ce document a été généré automatiquement le 1 septembre 2019

(c) École française de Rome 


\section{Cultes et pratiques rituelles chtoniens à Incoronata}

La campagne de 2018

\section{Mario Denti}

\section{NOTE DE L'AUTEUR}

Direction de la fouille : Mario Denti. Assistante : Emmanuelle Smirou (Laboratoire LAHM, UMR 6566 CReAAH, Université Rennes 2). Responsables de secteur : Josipa Mandić, Cesare Vita, Théo Ben Makhad, Chloé Damay (Laboratoire LAHM, UMR 6566 CReAAH, université Rennes 2). Responsable du laboratoire : Antonia Miola (Università della Basilicata). Responsable de la topographie et de la photogrammétrie : Théo Ben Makhad. Étude du mobilier : Clarisse Prêtre (UMR 7041 ARSCAN, Paris), Giulia Saltini Semerari (University of Michigan, Museum of Anthropology), Francesca Ippolito (University of Groningen). Laserscanner et relief drone : Vito Muscio (GeoNature. Geological and Geotechnical Consulting). Concession : Soprintendenza Archeologia Belle Arti e Paesaggio della Basilicata. Aide financière : Laboratoire LAHM, université Rennes 2. Centre Jean Bérard (Naples) - Ministère des Affaires étrangères et du Développement international (Paris).

1 La seizième campagne de fouille à Incoronata (qui a duré 5 semaines, de fin août à début octobre 2018) a été caractérisée par la valeur remarquable des structures et du mobilier mis au jour, permettant d'appréhender de manière presque définitive la configuration historique et fonctionnelle du site, occupé de la deuxième moitié du IX à la fin du VII ${ }^{\mathrm{e}}$ début du VI siècle $^{1}$.

2 L'exploration archéologique, menée sur le côté occidental de la colline en approfondissant et élargissant les secteurs fouillés dans les campagnes précédentes, a porté sur quatre contextes majeurs (fig. 1):

1. Le contexte cultuel chtonien du IX ${ }^{\mathrm{e}}$-VIII ${ }^{\mathrm{e}}$ siècle (secteur Sud);

2. L'espace contenant les deux petits fours du VII ${ }^{e}$ siècle (secteur Est) ; 
3. L'espace rituel du VII e siècle (secteur Nord) ;

4. De nouvelles structures monumentales (secteur Ouest).

3 Comme on le verra, le dénominateur commun de ces découvertes réside dans le fait que les espaces, les structures et les actions mêmes accomplies en relation à ces dernières ont été toutes, sans exception, réalisées dans la terre : creusements et aménagements du sol géologique pour y installer des structures à caractère rituel, en créant un véritable espace hypogé (1) ; creusement et aménagement du sol géologique pour y installer un espace associé à la pratique de la balanophagie (fours pour la torréfaction des glands) (2) ; creusements et aménagements de bothroi (3); construction de structures monumentales (murs, sols) installées à l'intérieur de grands espaces creusés dans le sol géologique (4).

\section{Le contexte cultuel chtonien du IX ${ }^{\mathrm{e}}-\mathrm{VIII}$ siècle (secteur Sud)}

4 Au sud du grand pavement en galets réalisé au VIII siècle (US 70, fig. 1, 2), qui longe toute la limite méridionale de cette partie de la colline, nous avons finalisé l'exploration des structures s'inscrivant à l'intérieur d'un espace aménagé directement dans le terrain géologique, qui a été artificiellement creusé et spécialement adapté pour les recevoir. La séance stratigraphique de cette zone - l'espace chronologiquement le plus ancien actuellement connu à Incoronata - a permis de reconnaître les phases et les actions suivantes :

1) La réalisation d'une grande fosse de forme subcirculaire (diamètre: $4,20 \times 3,10 \mathrm{~m}$; US 400 ; fig. 2,3 , B et 5 , ici partiellement fouillée), remplie par la superposition de différentes fines strates de terre noircie (fig. 3, C). Contenant des ossements d'animaux carbonisés et de la céramique décorée (urne avec triangles inscrits) et à impasto (coupe carénée à impasto noir lisse) datable de la deuxième moitié du $\mathrm{IX}^{\mathrm{e}}$ siècle ${ }^{2}$, ces couches apparaissent comme l'évident résultat d'une séance d'actions rituelles progressives. À noter que le bord de la fosse est soigneusement aménagé par des tapis de cailloutis et des plans en argile (fig. 3, A, D), lesquels ont été réalisés en succession, l'un sur l'autre, très probablement à l'occasion de chaque séance rituelle ${ }^{3}$. À l'intérieur, sur son côté occidental, une plus petite fosse de forme subcirculaire a été découverte (diamètre: $1,5 \times 1,2 \mathrm{~m}$; US 627 ; fig. 2 et 3, B); elle était vide.

2) La grande fosse a été ensuite recouverte par une dure strate de terre et de galets, qui l'a définitivement scellée (US 595, fig. 3, D). Sur cette strate a été construit un enclos constitué de trois alignements de pierres, formant un triangle parfaitement isocèle (côtés de $4 \mathrm{~m}$ environ en longueur), avec le sommet à l'est (fig. 2-4). Ce sommet correspond au lieu de l'aménagement rituel que nous avions mis au jour dans les campagnes précédentes, comprenant une grande pierre aniconique blanche (White Sandy Stone, fig. 3 , WS, 4, 5), interprétable comme un autel. Elle apparaissait entourée, au nord, par la déposition intentionnelle de gros galets (fig. 5 ), de céramique cérémonielle indigène, figuline et à impasto, datable du VIII siècle, et de nombreux ossements d'animaux ${ }^{4}$.

3) L'ensemble a été finalement oblitéré par un imposant recouvrement de grosses pierres informes, mélangées à une quantité remarquable de céramique réduite intentionnellement en fragments, comprenant, à côté de grands conteneurs, de la céramique à vocation cérémonielle de très haute qualité à «impasto liscio " (noir et noisette, datable encore du IX siècle) et des nombreuses ollai « a tenda " peintes et figurées, parfaitement reconstructibles ${ }^{5}$. 
Fig. 1 - Planimétrie de la fouille.

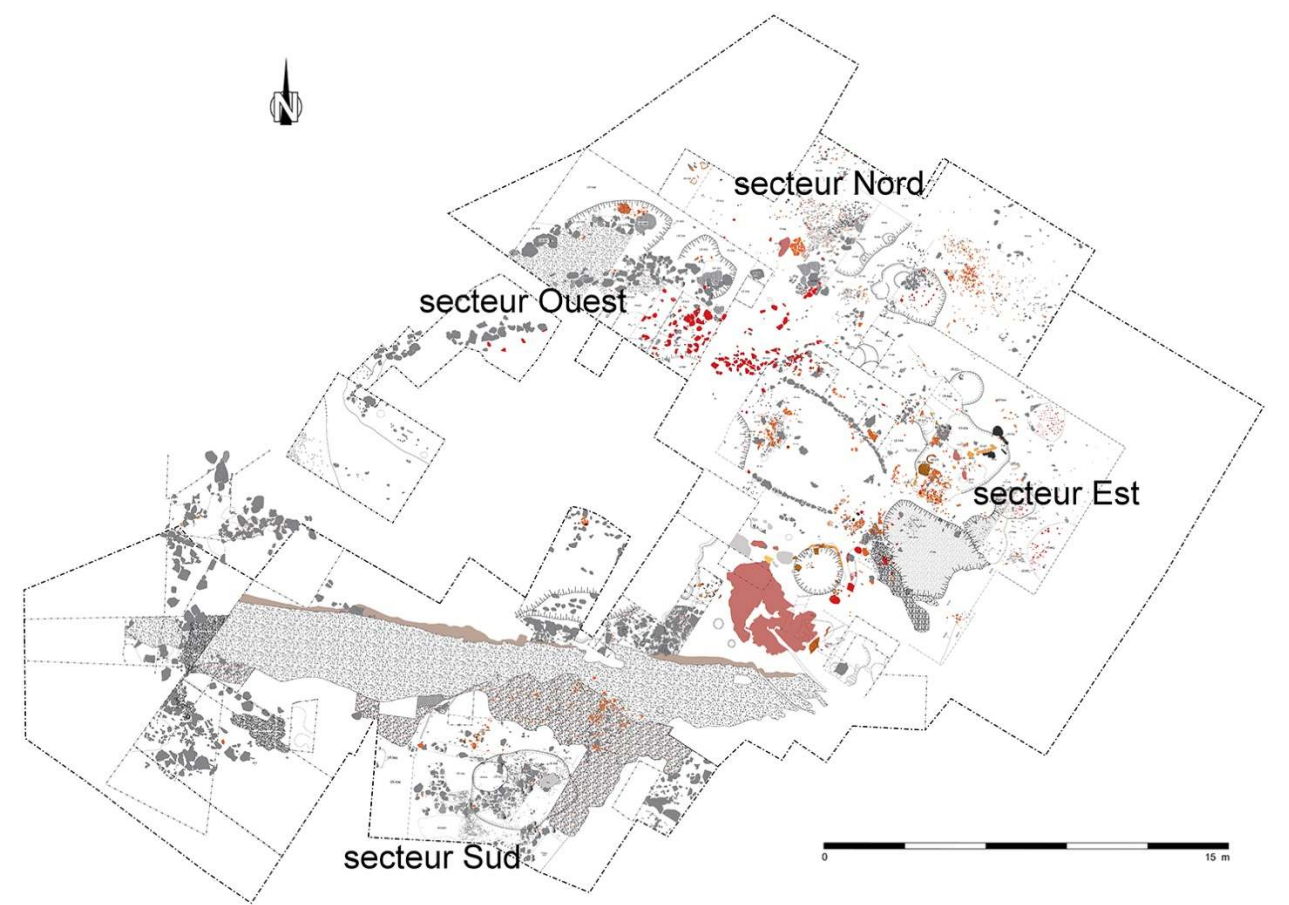

DAO T. Ben Makhad.

Fig. 2 - Planimétrie du secteur Sud : le contexte cultuel chtonien du IX ${ }^{\mathrm{e}}-\mathrm{VIII}{ }^{\mathrm{e}}$ siècle.

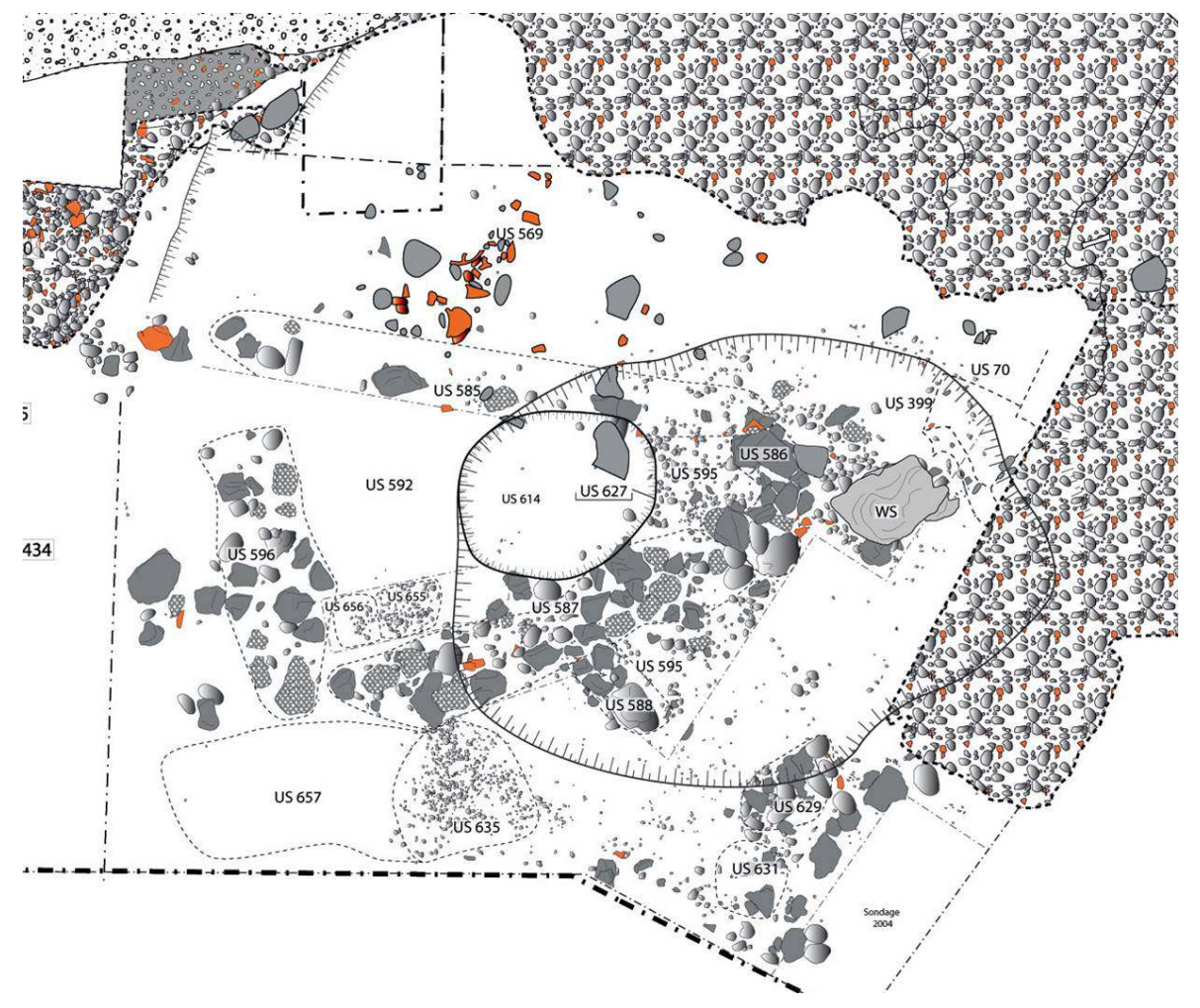

DAO T. Ben Makhad. 
Fig. 3 - Secteur Sud : le contexte cultuel chtonien du IX'-VIII' siècle, vu de l'est.

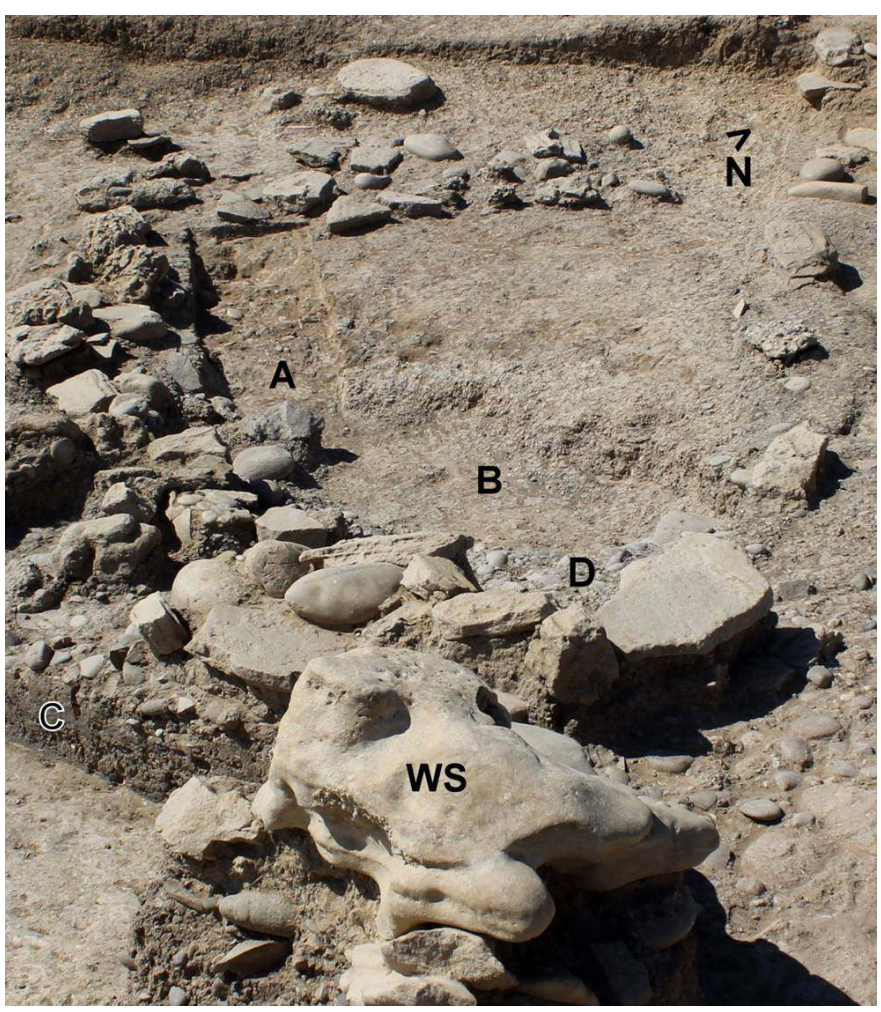

Cl. M. Denti.

Fig. 4 - Secteur Sud : le contexte cultuel chtonien du IXe-VIII' siècle, vu de l'ouest.

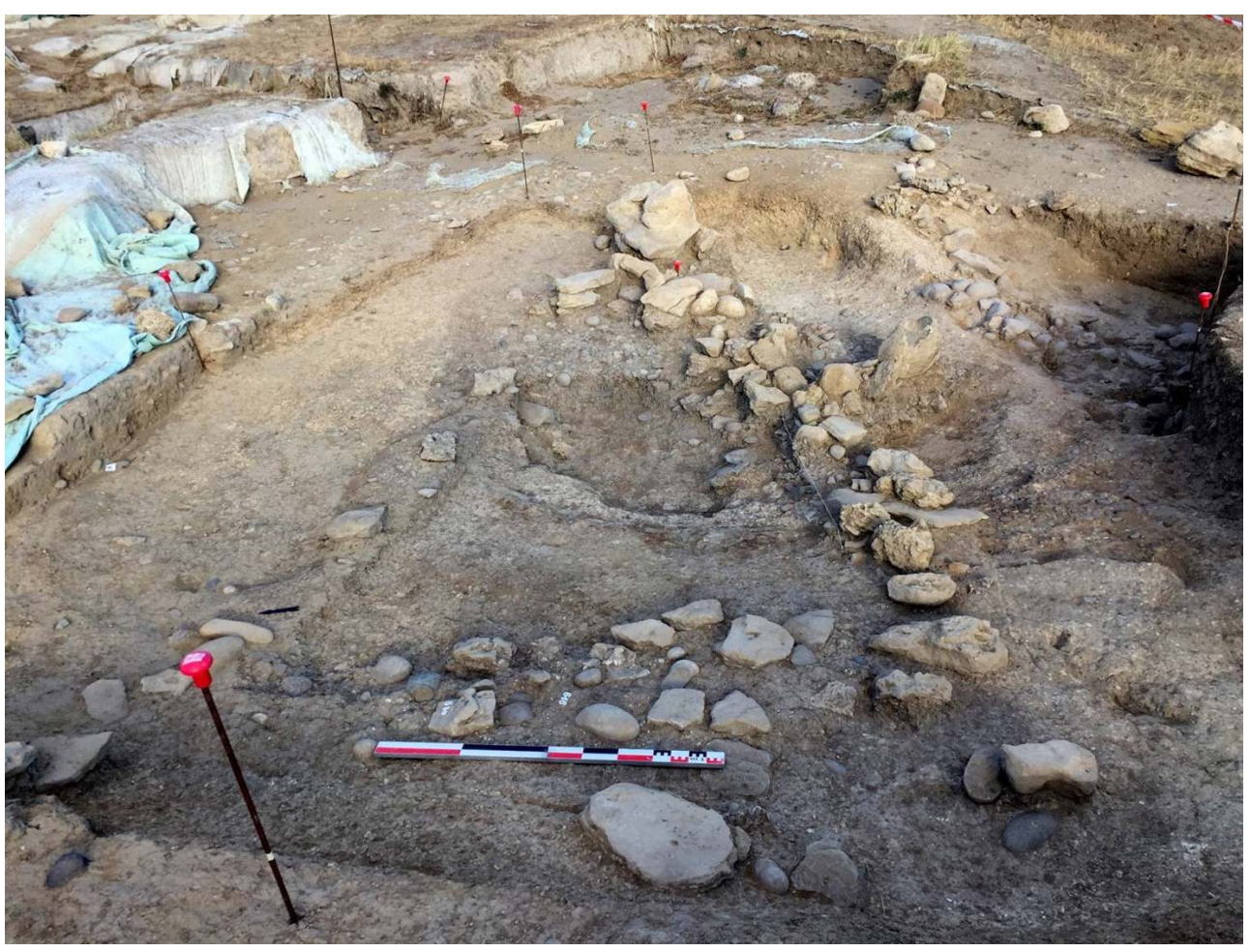

Cl. M. Denti. 
Fig. 5 - Secteur Sud : photogrammétrie du contexte cultuel chtonien, en cours de fouille (2017).

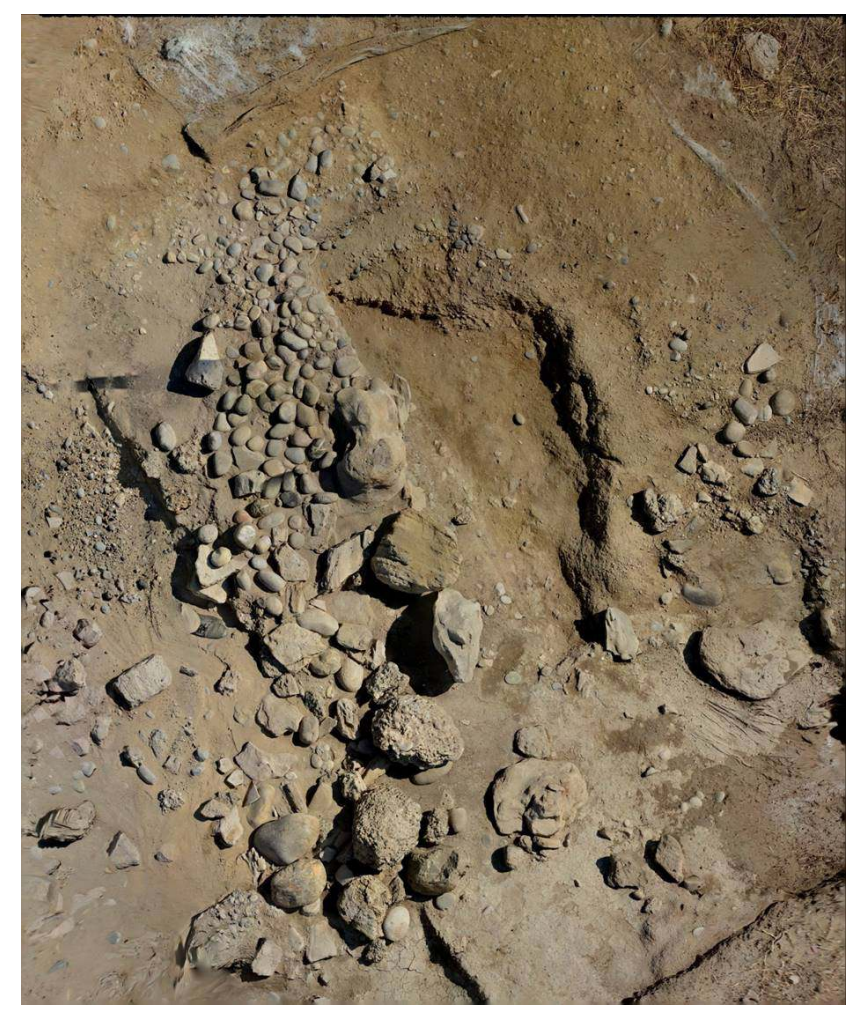

Élaboration T. Ben Makhad.

5 La succession de ces phases est datable entre la deuxième moitié du IX et le VIII ${ }^{\mathrm{e}}$ siècle, et se montre très cohérente sur le plan fonctionnel, en photographiant avec exactitude la séquence des actions rituelles accomplies dans un espace qui a dû jouer un rôle fondamental dans la plus ancienne configuration du site.

6 La forme triangulaire de l'aménagement en pierre, construit au-dessus de la grande fosse, une fois oblitérée, se révèle du plus grand intérêt. Elle renvoie directement à des structures de forme analogue, bien connues dans le monde égéen de l'âge du Fer, où elles sont constamment associées à des cultes de type héroïque ${ }^{6}$. La découverte, l'année dernière, d'une déposition de perles de verre, lapis-lazuli et clous en bronze, à côté de cet aménagement ${ }^{7}$, avait déjà pu nous orienter vers une contextualisation funéraire des actions rituelles ici attestées.

7 Si l'extension de la fouille dans la prochaine campagne permettra de confirmer cette tournure interprétative, les trois séances actuellement reconnues pourraient bien correspondre au schéma suivant (à retenir, bien évidemment, comme pure hypothèse de travail) :

1) Située au sein de la grande fosse rituelle US 400, la plus petite fosse (US 627), retrouvée vide, pourrait hypothétiquement constituer le creusement d'une tombe, justement en raison du fait qu'elle a été trouvée vidée 8 . Ce serait en relation à cette dernière, donc, qu'une série d'actions rituelles a été effectuée à l'intérieur de la grande fosse. Celles-ci comprenaient des sacrifices, comme l'indique l'alternance de couches de terre noire, contenant de la céramique noircie, des os carbonisées, des charbons de bois (fig. 3, C).

2) Au moment de l'oblitération de cet espace cultuel chtonien, la « tombe » US 627 aurait été vidée, la fosse US 400 remplie et scellée par la strate de galets US 595, et l'enclos 
triangulaire construit par-dessus, en guise de véritable cénotaphe. À l'extrémité est de l'enclos triangulaire, exactement en correspondance de son angle oriental, un autel en forme de pierre aniconique (fig. 3,WS) a été aménagé, et des pratiques rituelles, comprenant des libations et des repas, ont été réalisées tout autour (déposition de céramiques et d'os d'animaux, fig. 5). Si cette interprétation devait se confirmer, ce ne serait pas inconcevable de pouvoir retrouver, une fois la fouille élargie, d'autres fosses (vides ou non) à côté de cet enclos, témoignant de la couleur chtonienne de ces cultes : l'articulation enclos triangulaire/tombes, documentée dans l'exemple le mieux étudié, Érétrie ${ }^{9}$, suggère en effet cette éventuelle piste interprétative.

3) Tout cet espace a été finalement oblitéré par un imposant recouvrement de pierres (provenant, très probablement, de la démolition partiale de l'enclos et d'autres possibles structures environnantes) mélangées à de la céramique intentionnellement fragmentée. Il n'apparaît pas encore clairement si cette oblitération a été réalisée au même moment ou bien en deux phases: l'une relative à la fermeture de l'enclos, l'autre relative à la fermeture de l'espace rituel de l'« autel » WS ${ }^{10}$.

8 Au-delà du possible degré d'exactitude de cette hypothèse reconstructive - certainement à vérifier avec la poursuite de nos recherches - il est important de souligner la dimension de cette découverte : si d'un côté elle permet de confirmer la couleur fondamentalement rituelle de la plus ancienne phase d'occupation de notre colline, de l'autre, elle ouvre de perspectives majeures et nouvelles dans l'archéologie du culte des communautés indigènes de l'âge du Fer en Italie méridionale, en soulignant tout le poids de la problématique de leur plus ample inscription dans l'horizon politique et culturel méditerranéen.

\section{L'espace contenant les deux petits fours du VII'e siècle (secteur Est)}

9 À côté de l'angle nord-oriental de l'entrée de l'édifice absidé (fig. 1), la poursuite de l'exploration de la zone où deux petits fours avaient été mis au jour l'année dernière ( fig. 6-7) a permis de qualifier de manière plus précise la fonction de ce contexte. Nous avons en effet mis au jour un espace délimité par un creusement peu profond du sol géologique (d'une vingtaine de $\mathrm{cm}$ : fig. 7), dont les limites extérieures sont connectées à un plan qui correspond stratigraphiquement à celui sur lequel gisent les pierres constituant le périmètre de l'édifice absidé. Cette circonstance permet de confirmer que les deux structures ont fonctionné ensemble.

10 Cet espace, datable du VII ${ }^{e}$ siècle, est conservé en parfait état, puisque non seulement il a été scellé par des strates d'oblitération - et par un sol en cailloutis qui recouvraient spécialement la partie correspondant aux deux petits fours ${ }^{11}$ - mais aussi, car, au moment de son abandon, il a subi une très importante action du feu, sans laquelle les restes botaniques découverts (si remarquables, comme on le verra) n'auraient pas pu être conservés. L'espace est caractérisé par de considérables concentrations de céramique (notamment de cuisine, fragmentée mais parfaitement recomposable) et de larges amas d'argile rubéfiée et noircie. Sur son côté occidental, il y a deux petits fours, conservés dans un état de conservation exceptionnel (fig. 7-8). Ils sont adjacents l'un à l'autre et présentent le praefurnium ouvert à l'est (mesures des diamètres : $57 \times 43$ et $30 \times 25 \mathrm{~cm}$ ). Leur orientation correspond à celle de l'édifice absidé adjacent: l'axe sur lequel les 
bouches des fours sont posées est presque le même que l'axe de l'entrée du bâtiment. Toutes ces structures sont, en effet, orientées à l'est (fig. 6). Les petits fours ont les parois, de forme ovale, réalisés en argile. Le four le plus grand est caractérisé par la présence d'un fond plat en argile (fig. 10), absent de l'autre (fig. 7-8).

Leur état de conservation est dû au fait qu'ils ont été soigneusement oblitérés. Après avoir été sectionnés en horizontal, en enlevant leur coupole, leur fragiles parois ont été consolidées par l'insertion, à leur intérieur, de morceaux d'adobe et de sole appartenant à des fours de dimensions bien plus grandes (fig. 9). Juste devant le praefurnium du four le plus grand, nous avons trouvé des anneaux en bronze et, sur son côté ouest, un skyphos de production grecque locale (fig. 9). Ces dépositions confirment la couleur rituelle de la défonctionnalisation de ce contexte, qui a prévu une pratique libatoire, témoignée par la coupe. Autour (fig. 8) comme à l'intérieur (fig. 10) des deux fours se trouvaient des dizaines de glands de chêne, parfaitement conservés grâce à l'action du feu, qui les a complètement séchés et carbonisés. Ils apparaissent souvent directement associés aux restes de leurs récipients, des conteneurs à impasto, achromes ou monochromes, de taille moyenne (fig. 8).

Fig. 6 - Secteur Est : planimétrie de la zone de l'espace contenant les deux petits fours.

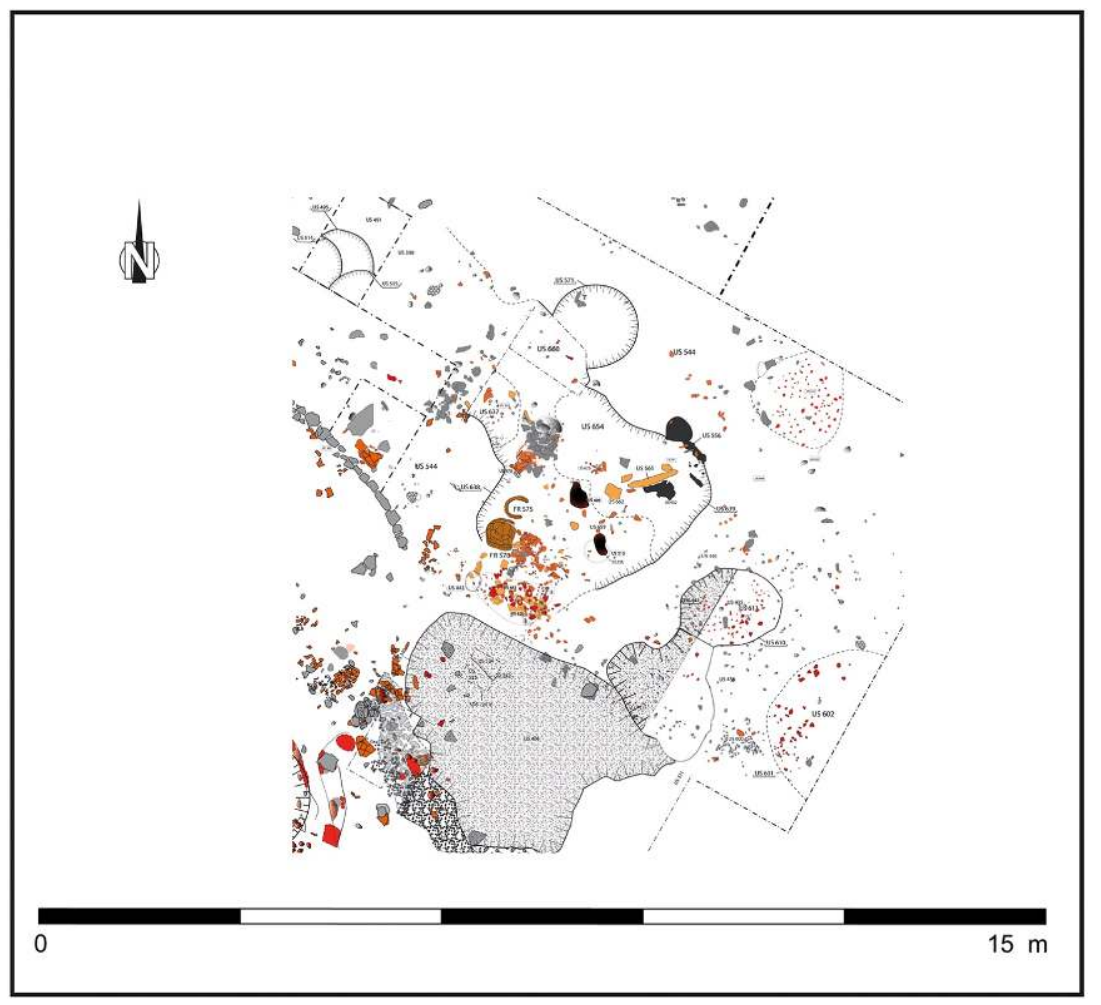

DAO T. Ben Makhad. 
Fig. 7 -Secteur Est : l'espace contenant les deux petits fours, vu du nord.

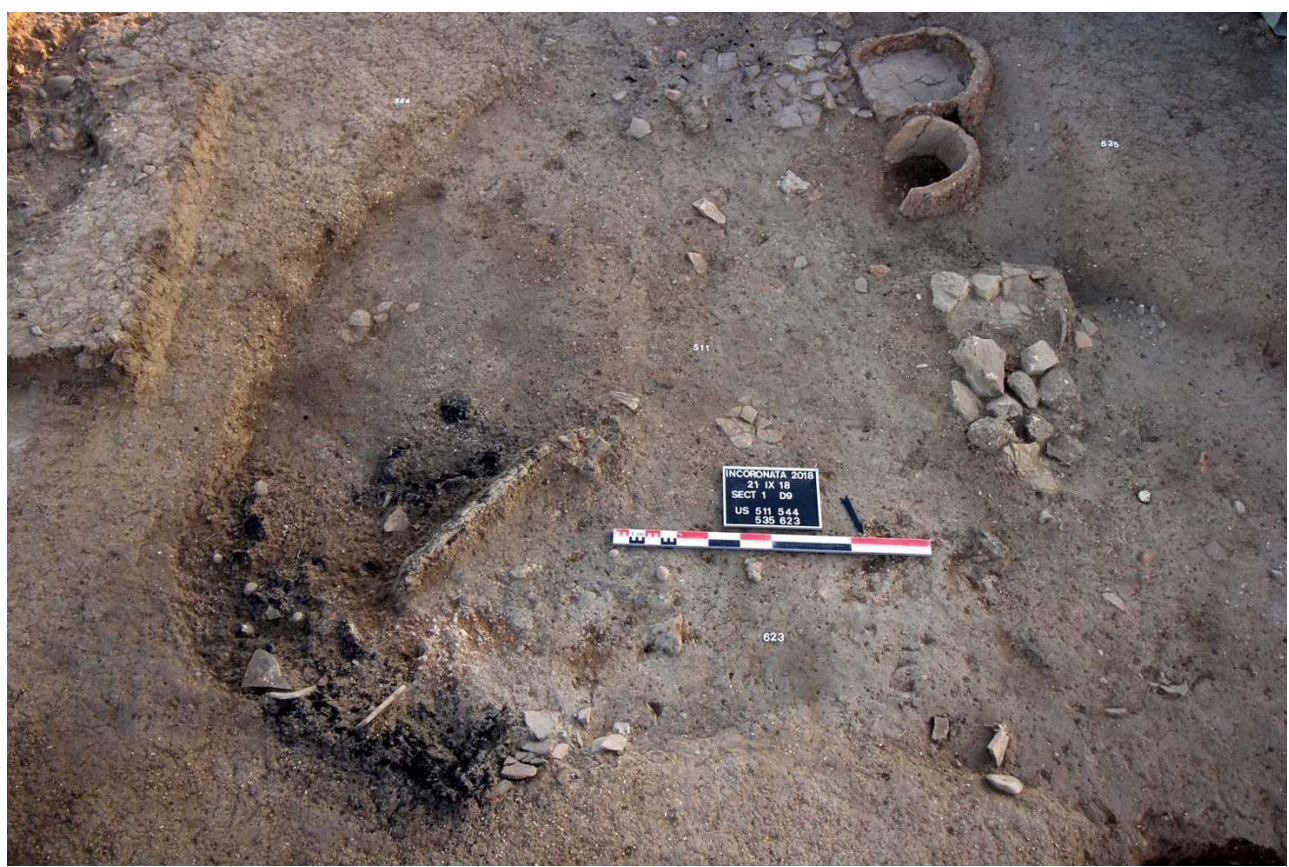

Cl. M. Denti.

Fig. 8 -Secteur Est : les deux petits fours, les concentrations des céramiques, les glands et, à droite de l'image, la grande olla à impasto avec la petite structure en pierres US 492.

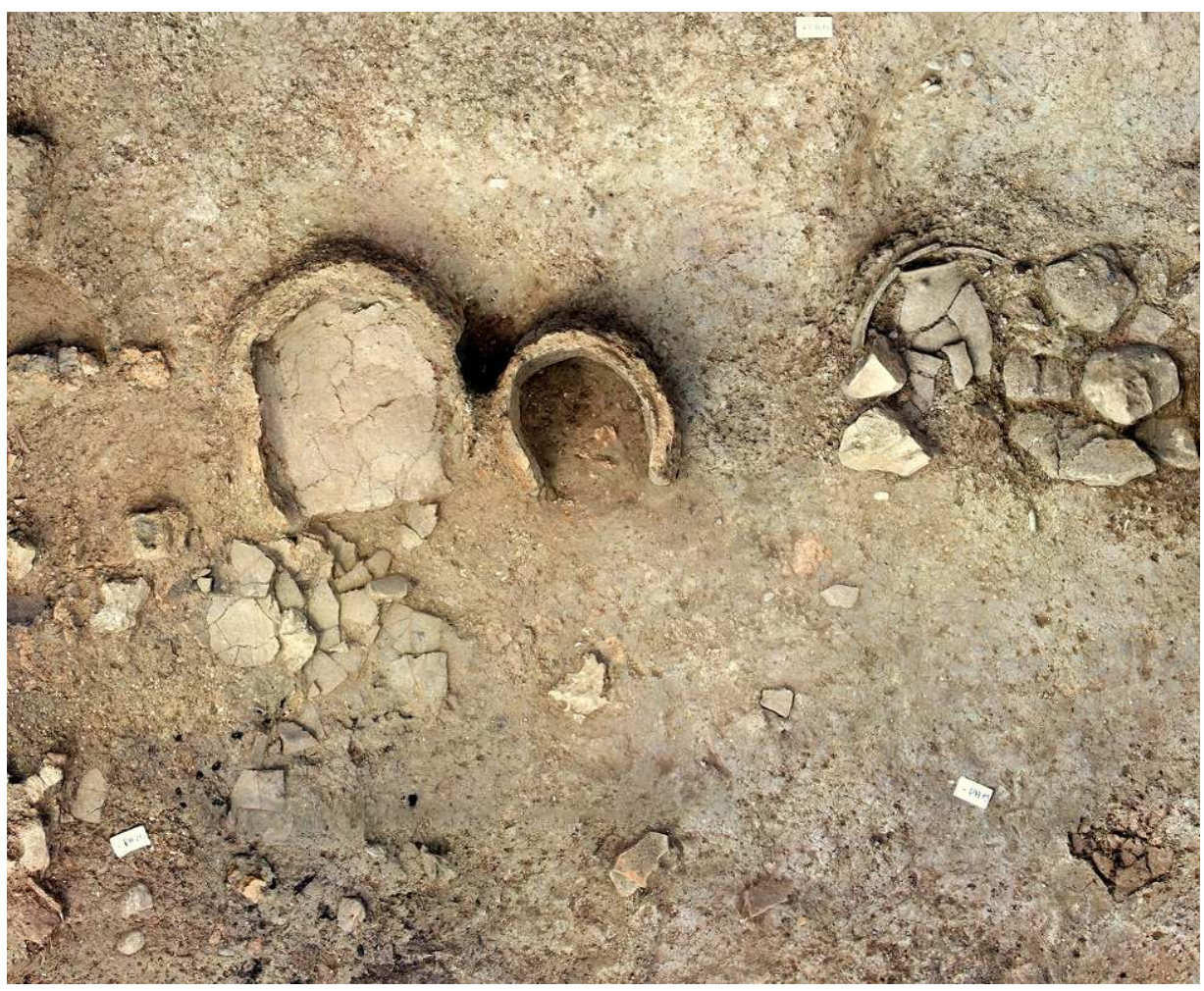

Cl. M. Denti. 
Fig. 9 - Secteur Est : les deux petits fours, encore remplis de matériel oblitérant, avec la paroi d'un skyphos déposée sur l'angle occidental du four le plus grand.

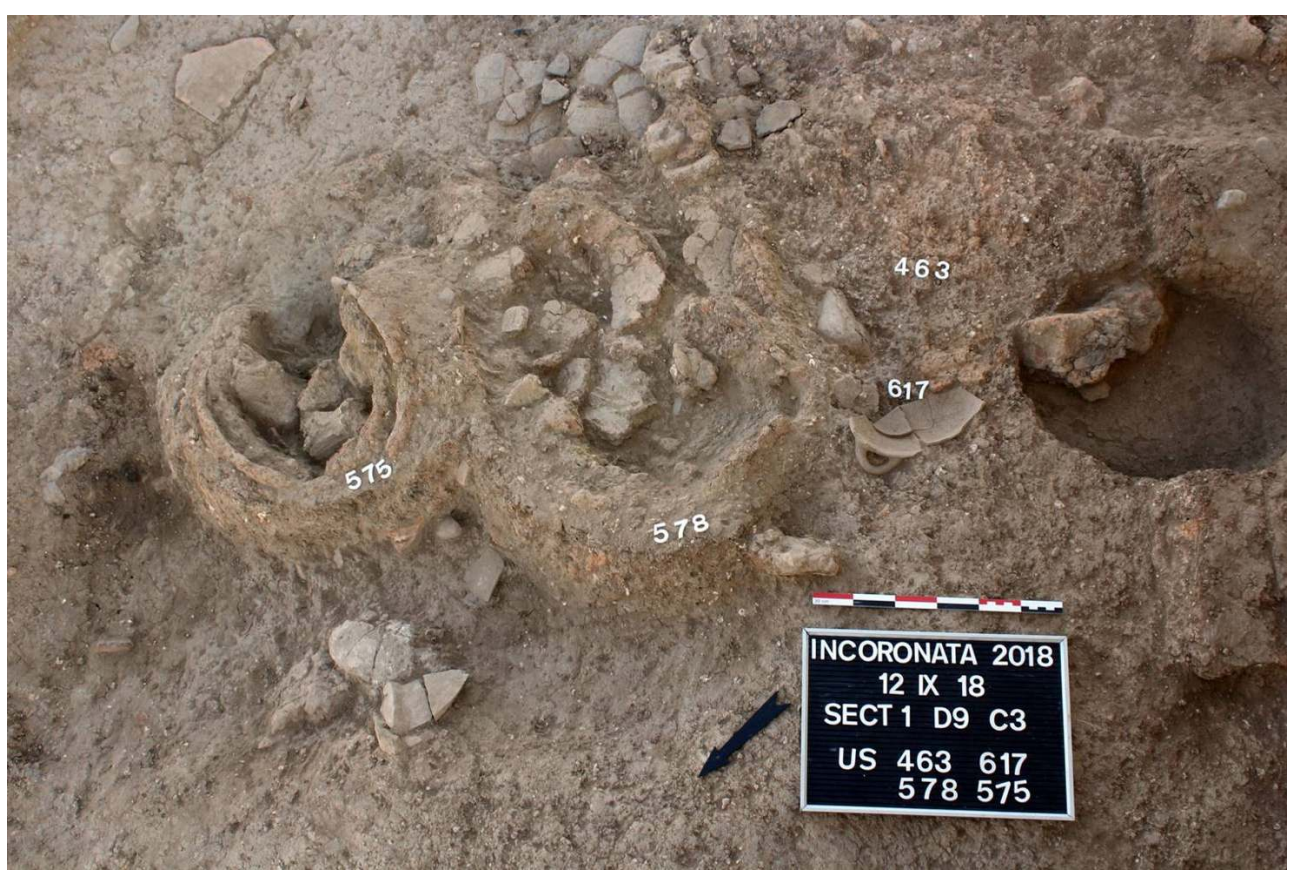

Cl. M. Denti.

Fig. 10 - Secteur Est : les glands à l'extérieur et à l'intérieur des parois de l'un des petits fours.

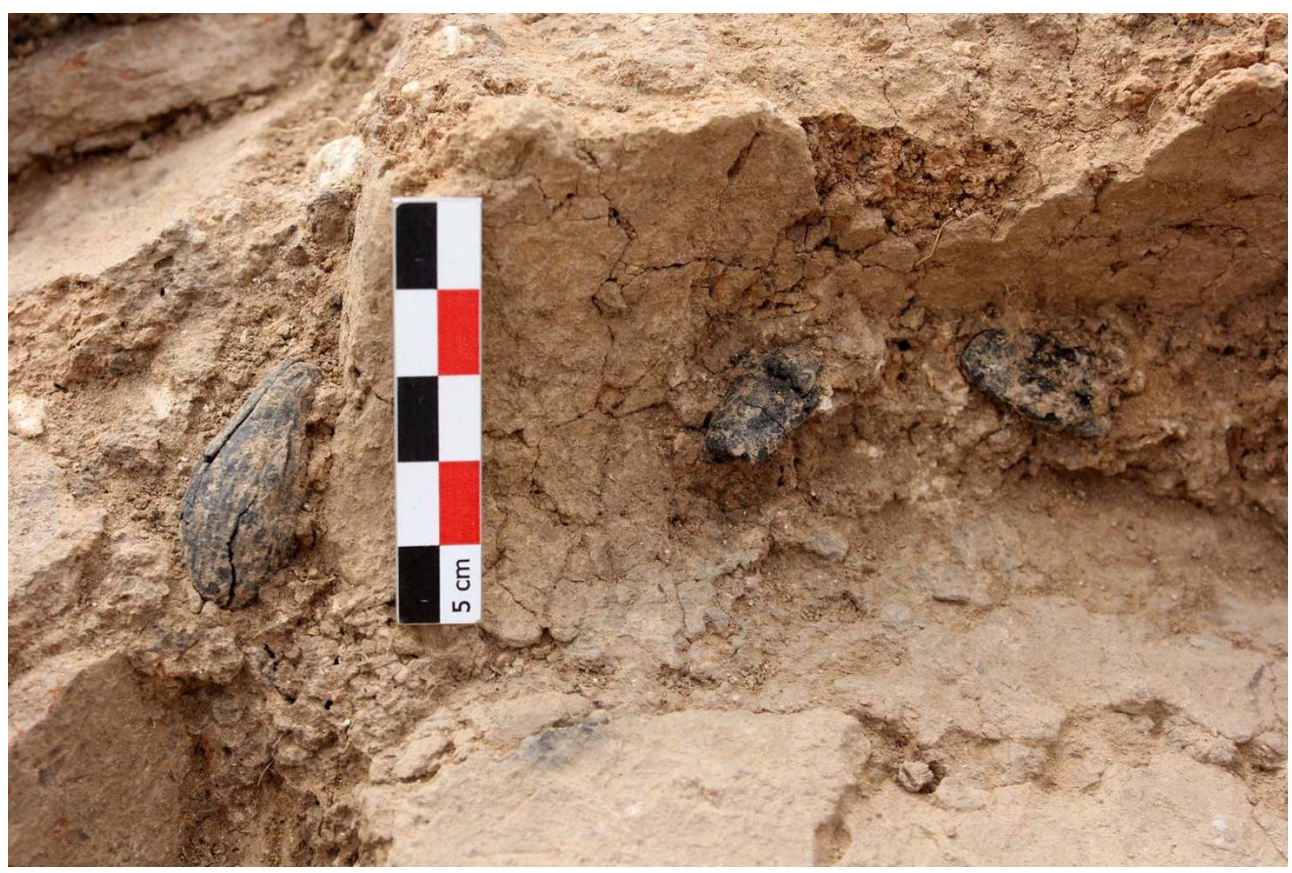

Cl. M. Denti.

12 Juste au Nord des petits fours, un plan - probablement de cuisson - a été mis au jour. Il est constitué de tessons de céramique indigène (achrome et monochrome) aménagés à plat (US 636, fig. 6-8), entourés par la paroi d'un grande olla à impasto d'un côté, et d'un 
autre par une petite structure en pierres (US 492). Le tout était recouvert par la déposition de 4 pesons, dont 3 décorées avec des motifs incisés, et d'une fusaïole (fig. 11).

Fig. 11 -Secteur Est : fusaïole et pesons, dont 3 décorés, déposés au-dessus de la petite structure en pierres US 492.

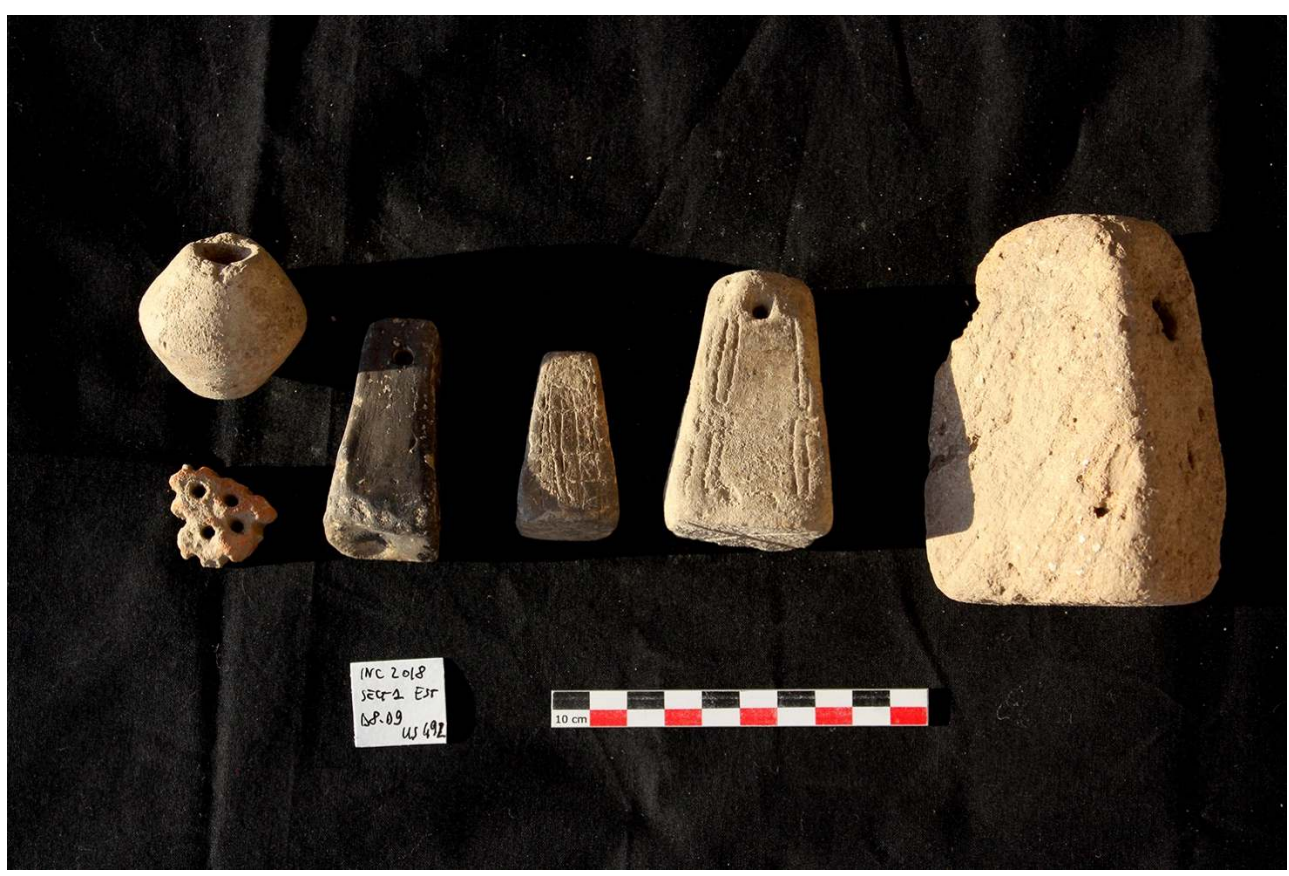

Cl. M. Denti.

13 Une fosse de forme parfaitement circulaire (diamètre: 1,10 $\mathrm{m}$; profondeur : $28 \mathrm{~cm}$ ) s'ouvre sur le côté nord de cet espace (fig. 6). Elle a été remplie de terre foncée, contenant des petits charbons et de la céramique, notamment monochrome et bichrome, mais aussi grecque, et une grosse paroi d'un pithos. Appuyée sur la limite nord-orientale du creusement qui délimite cet espace, une remarquable concentration de gros tronçons de bois carbonisés a été mise au jour (fig. 6-7). Les tronçons sont conservés dans un état de conservation exceptionnel et, au regard du système d'assemblage observé entre eux, ils pourraient appartenir à un gros objet de forme rectangulaire (fig. 12). 


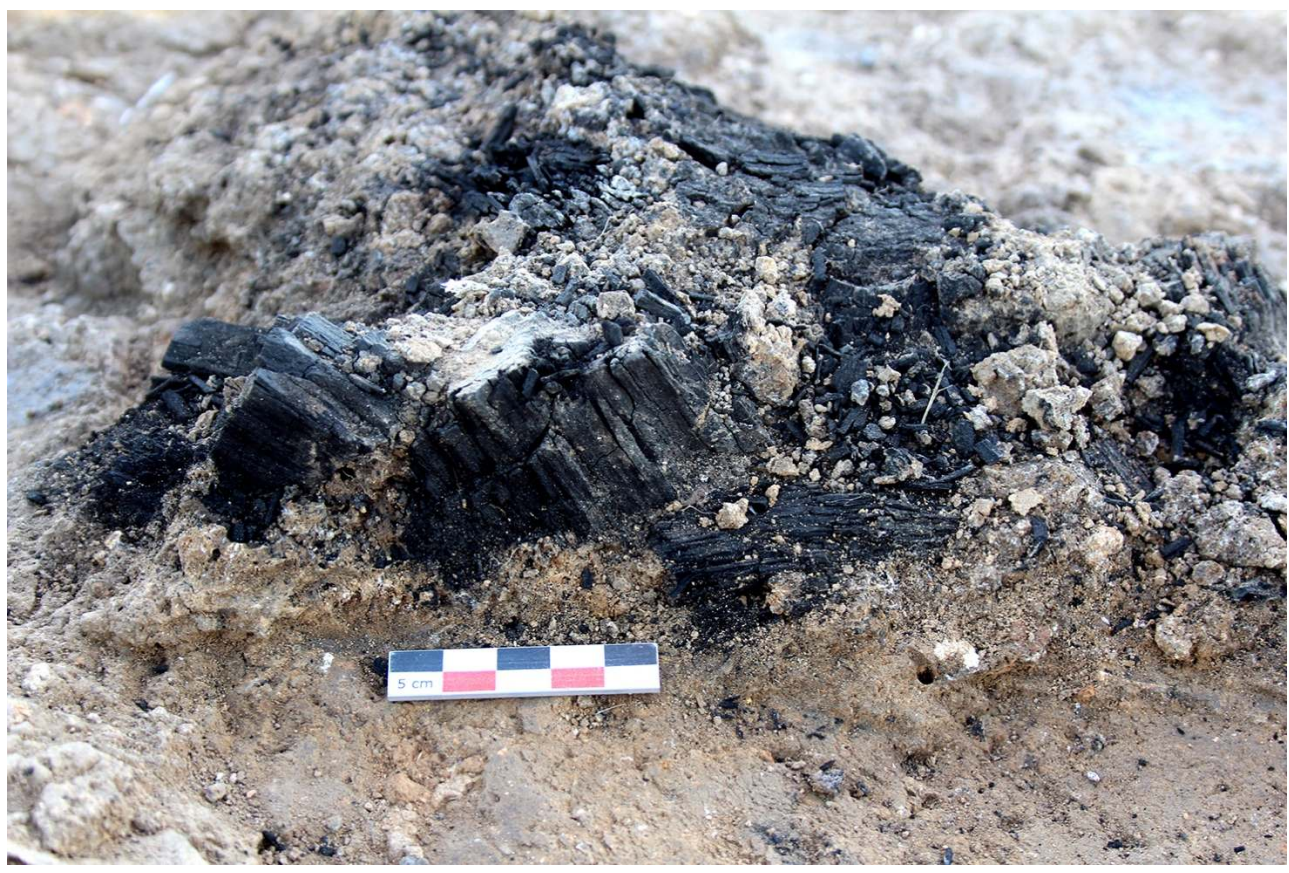

Cl. M. Denti.

L'état de conservation de cette documentation a rendu nécessaire l'ablation de l'ensemble des éléments en bois et des deux petits fours. Ces éléments ont été ainsi enlevés en utilisant la technique du «strappo », par un spécialiste de la Soprintendenza Archeologia Belle Arti e Paesaggio della Basilicata, et amenés dans les réserves du Museo Archeologico Nazionale di Metaponto, où ils feront l'objet d'études spécifiques. Les glands, la terre dans laquelle ils ont été retrouvés et les éléments en bois, feront l'objet d'analyses menées par le Laboratorio di Archeobotanica de l'université de Lecce. La cohérence documentaire de ce contexte, impliquant une association étroite entre fours, céramiques et glands, permet d'en proposer une lecture comme espace destiné à la torréfaction des glands pour un usage alimentaire. La balanophagie est d'ailleurs une pratique amplement pratiquée en Italie méridionale à partir de la moitié du deuxième millénaire, et notamment dans la partie orientale de la péninsule, où elle est bien attestée dans des espaces domestiques mais également dans des espaces sacrés (on rappellera en particulier le cas de Roca Vecchia $)^{12}$.

15 La modalité de l'oblitération ritualisée de notre espace, incluant la fragmentation intentionnelle de la céramique, la déposition d'au moins un skyphos, d'éléments en bronze associés aux deux fours et d'une série de pesons décorés, aussi bien que le recours à l'action purificatrice du feu, suggèrent - à côté d'une fonction domestique - une possible destination cultuelle de cette activité. Cette destination se révèle par ailleurs particulièrement cohérente par rapport à la couleur rituelle de l'ensemble de la zone qui entoure l'édifice absidé adjacent, à l'intérieur duquel des cérémonies à caractère chtonien avaient lieu : il s'agit en effet d'une zone caractérisée par la présence de nombreux bothroi , d'un autel et d'une série de contextes dépositionnels, datables à la phase chronologique gréco-indigène du VII ${ }^{e}$ siècle. Nous connaissons du reste bien les profondes liaisons conceptuelles entretenues, dans l'Antiquité, entre le chêne - arbre oraculaire connecté au culte des ancêtres - et les rites à caractère chtonien ${ }^{13}$. Finalement, il faut rappeler 
qu'activités domestiques et activités rituelles ne sont pas deux sphères dichotomiques dans les témoignages archéologiques relatifs à la consommation des céréales dans l'Antiquité : celle-ci, en effet, pouvait recouvrir à la fois des fonctions alimentaires, diététiques, symboliques et cérémonielles ${ }^{14}$.

\section{L'espace rituel du VII siècle (secteur Nord)}

$16 \mathrm{Au}$ nord de l'édifice absidé (fig. 1), nous avons poursuivi la fouille d'une partie du contexte rituel, datable du VII siècle, comprenant un ample aménagement de galets de petites dimensions régulières (US 526), posés à plat directement sur la terre sur plusieurs niveaux, sur lesquels gisait une remarquable quantité d'ossements d'animaux et de tessons appartenant pour la plupart à des skyphoi de production locale (fig. 13). Cet aménagement - un véritable tapis de galets - entoure une fosse (US 582) que nous avions fouillée l'année dernière seulement sur un quart et interprétée comme bothros, remplie par de la terre cendreuse contenant du charbon de bois, des os et de la céramique ${ }^{15}$. Ce contexte, où des pratiques rituelles avaient entraîné la consommation de la viande et du vin, est d'ailleurs directement associé à un autel de pierres (US 381), qui s'élève juste sur son côté sud (fig. 13) ${ }^{16}$.

Fig. 13 - Secteur Nord : l'aménagement galets-fosse US 582 vu du Nord, à la fin de la fouille, avec l'autel de pierres (US 381) au sud, en haut de l'image.

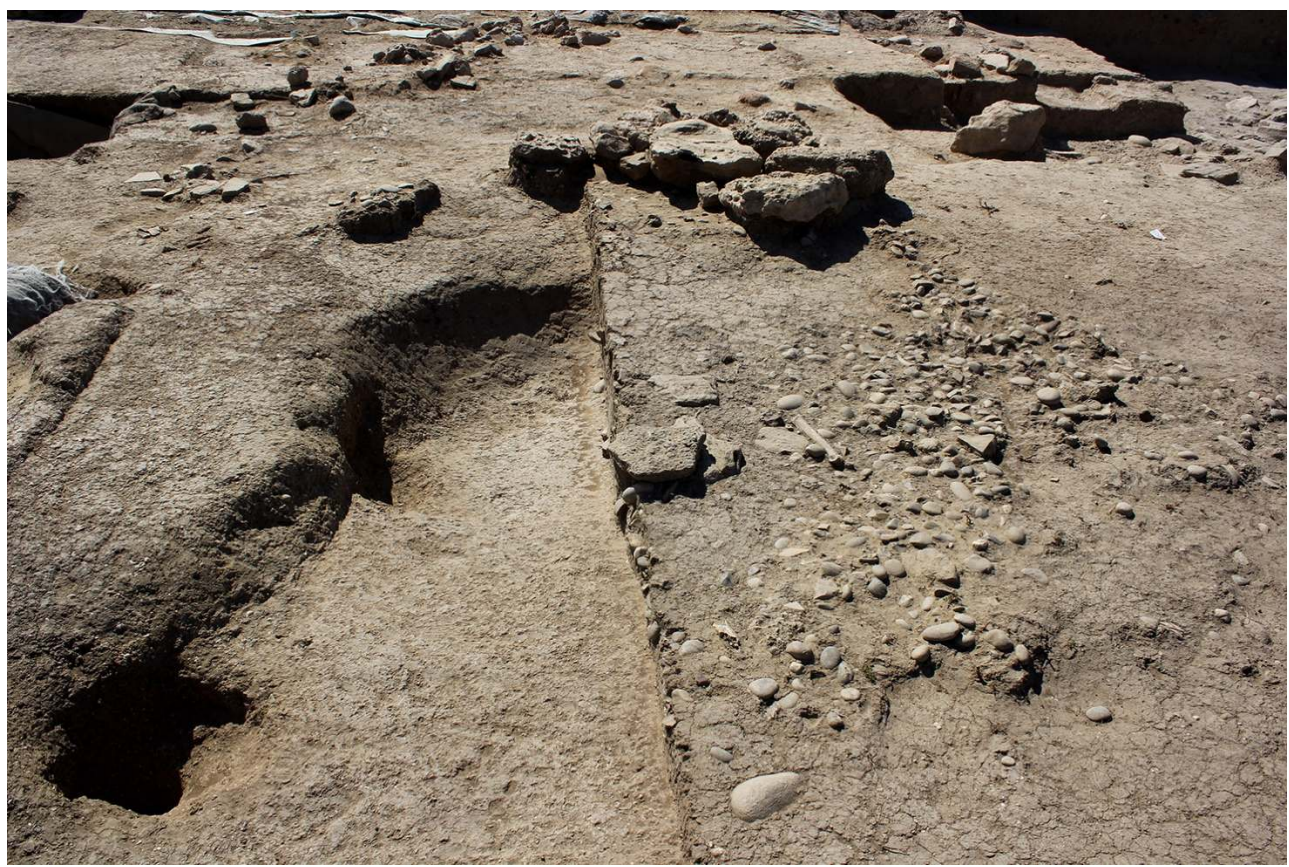

Cl. M. Denti.

Le sondage de cette année, réalisé dans le coté est du secteur, a permis d'intercepter le profil septentrional et le fond de ce bothros, mais surtout d'observer une particularité très significative pour la connaissance du déroulement des procédures rituelles de type chtonien. La limite de l'agencement du plan de petits galets, qui entoure au nord le bothros, s'interrompt en effet précisément en correspondance de la partie de la fosse dans laquelle le remplissage contient de la céramique. Au contraire, les galets recouvrent toute la partie de la fosse où la déposition de céramique est absente. Cela signifie - comme nous 
avons pu souvent l'observer à Incoronata - que l'aménagement de galets est installé pour délimiter l'espace de la pratique rituelle : il marque visuellement sur terre ce qui se passe sous terre ${ }^{17}$.

\section{De nouvelles structures monumentales (secteur Ouest)}

18 Au nord-ouest de l'édifice absidé, à la limite du plateau collinaire (fig. 1), nous avons poursuivi l'enlèvement de grandes couches d'oblitération constituées au cas par cas de terre, pierres, écailles et briques, qui caractérisent de manière impressionnante tout ce secteur de la colline. Leur présence nous avait déjà permis de détecter l'existence d'éléments et de structures sous-jacentes - des structures qui avaient été retenues justement dignes de recevoir une oblitération de telle envergure - et de localiser parfois à l'avance, grâce à l'observation de la mise en place de strates de terre de couleurs et consistances différenciées, les limites mêmes de ces structures. Ainsi, un énorme tas de terre extrêmement compact et de couleur rougeâtre, contenant des centaines de briques intentionnellement fragmentées (US 453, fig. 14, déjà en partie fouillé l'année dernière ${ }^{18}$ ), avait été réalisé au-dessus d'une série de structures du plus grand intérêt : celles-ci ont été démolies, gardées au niveau de la fondation, et finalement oblitérées sous cet énorme recouvrement.

Fig. 14 - Secteur Ouest (vu de l'est) : les structures monumentales émergeant, en cours de fouille, sous la grande couche d'oblitération remplie de briques US 453 .

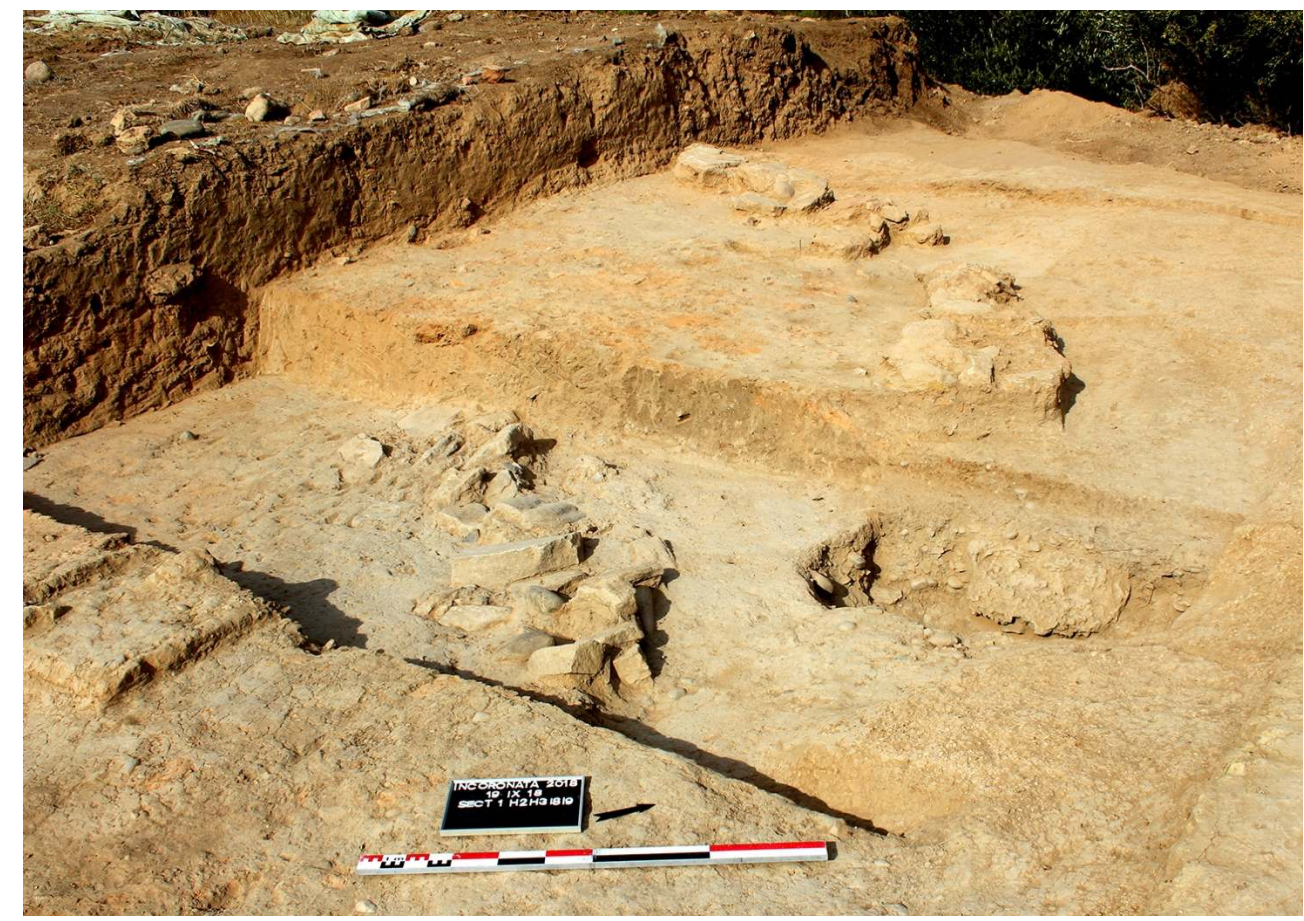

Cl. M. Denti.

Installées à l'intérieur d'un grand creusement réalisé dans le terrain géologique ( fig. 15-16), ces structures comprennent un sol en fins cailloutis (US 640), délimité au nord par des amas de terre soutenant des pierres à plat (US 618). Celles-ci longeaient presque 
parallèlement le profil du creusement, en construisant une sorte de démarcation avec une orientation semi-circulaire (US 618, fig. 14-17). Le sol, retrouvé en parfait état de conservation, est associé à une grande structure en pierres informes (largeur: 1,5 m environ, US 591), qui semble s'étaler vers l'est dans le même sens (semi-circulaire) de la limite septentrionale du sol (fig. 15-16). Pourrait-il s'agir d'un niveau de fondation?

Fig. 15 - Planimétrie du secteur Ouest.

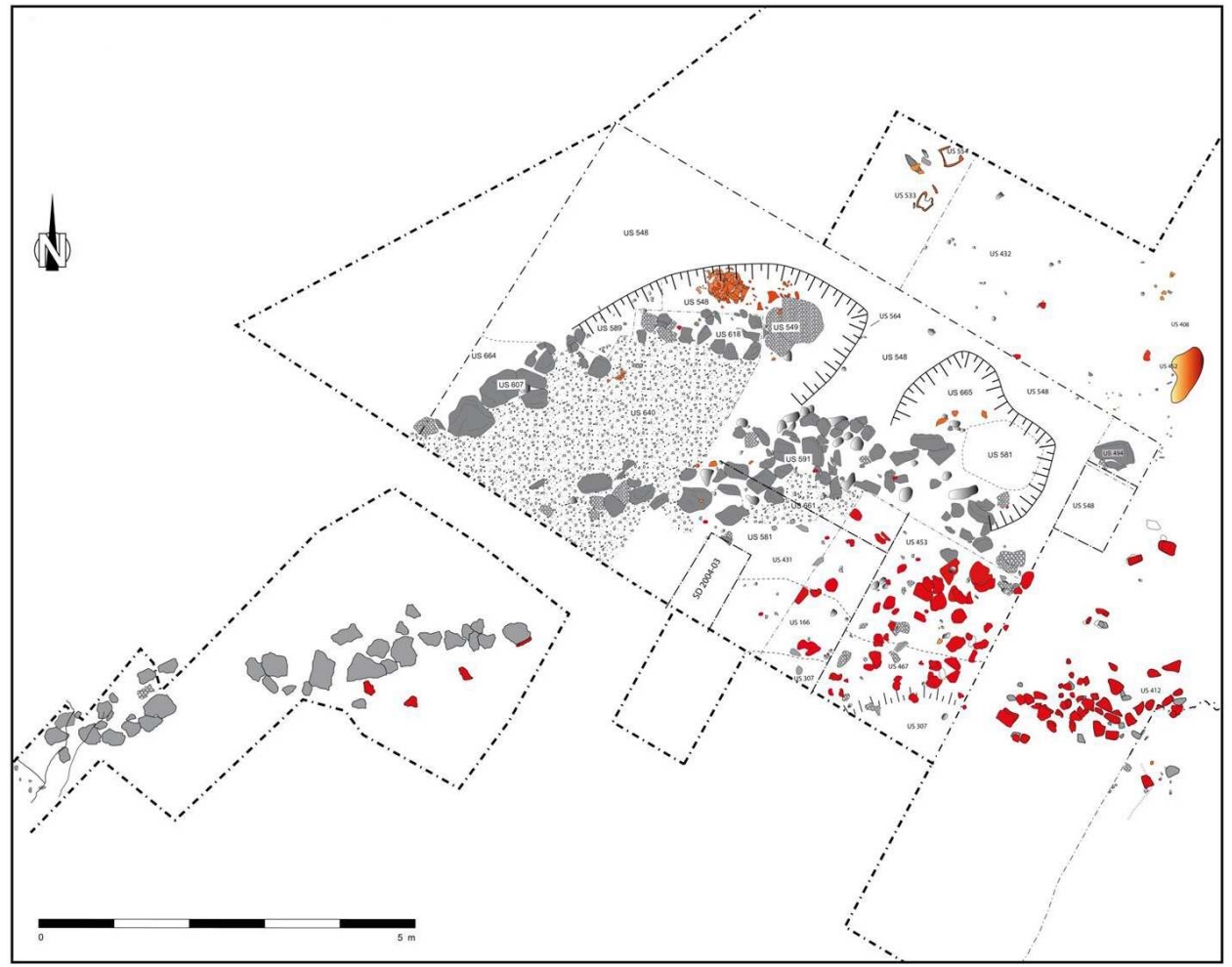

DAO T. Ben Makhad. 
Fig. 16 - Secteur Ouest, vu de l'est.

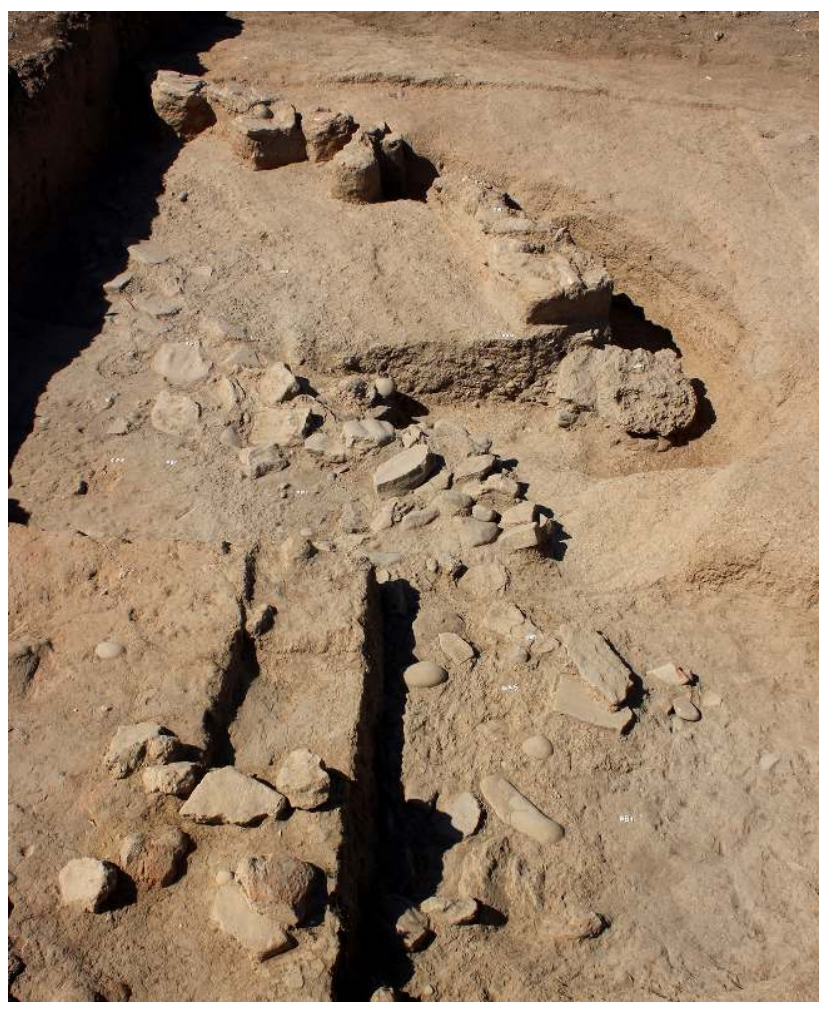

En premier plan, les couches d'oblitération; au centre, la structure en pierres US 591 et le sol US 640, en cours de fouille ; vers le nord, la délimitation de ce dernier, US 618, qui suit le profil incurvé du croisement dans le sol géologique.

Cl. M. Denti. 
Fig. 17 - Secteur Ouest, vu du nord.

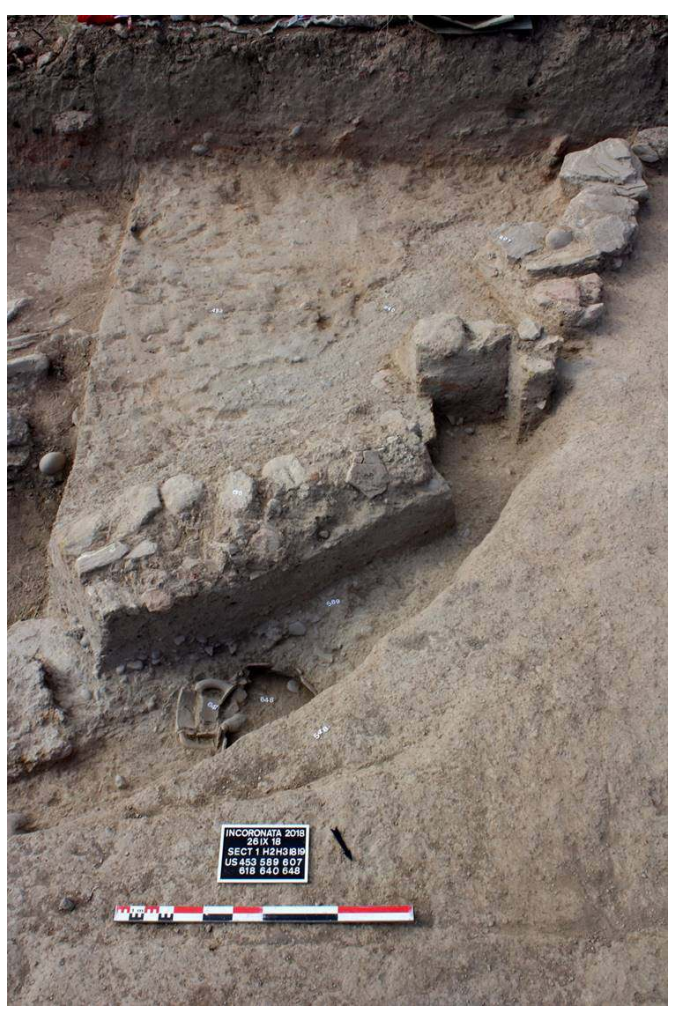

La limite septentrionale du sol US 640, avec la fosse de déposition réalisée entre cette dernière et le creusement du sol géologique, où est bien visible l'amphore corinthienne.

Cl. M. Denti. appartenir à un même bâtiment, dont nous ne connaissons pour l'heure ni les limites ni la configuration (que nous comptons intercepter dans la campagne de 2019). Les briques rejetées dans la couche d'oblitération auraient-elles pu appartenir à l'élévation de cette structure si monumentale? Si oui, cela signifie que les grandes briques d'Incoronata - il s'agit de briques cuites, mesurant $30 \times 40 \times 8-9 \mathrm{~cm}^{19}$ - auraient finalement retrouvé (l'un de) leur édifice d'appartenance.

21 Au niveau du mobilier, une série de découvertes majeures a permis de commencer à déterminer la fonction de ce bâtiment. À l'intérieur de la couche d'oblitération qui se trouve au nord du sol (fig. 17) - longeant le creusement entaillé dans le terrain géologique, qui devait alors se trouver en dehors du bâtiment, au-delà de sa limite septentrionale - une déposition de céramique a été mise au jour (fig. 15, 17). À côté d'une quantité remarquable d'os d'animaux, elle inclut de la céramique presque exclusivement grecque, avec quelques rares tessons indigènes dans les niveaux supérieurs. Il faut souligner qu'il s'agit de céramique d'importation, majoritairement de fabrication corinthienne, de très haut niveau d'exécution, et extrêmement cohérente en termes de fonction (fig. 18). La panoplie entière de la libation et de l'offrande sacrificielle y est attestée: parmi d'autres objets, nous citerons une amphore corinthienne de type A, déposée entière (fig. 19); des parois d'une amphore SOS ; des parties d'une œnochoé du PC Ancien ou Moyen (fig. 20); de très nombreuses coupes, skyphoi et kotylai PC (dont un avec un graffito, fig. 18); un krateriskos PC (fig. 21); des parties d'un grand louterion; des 
parties d'une chytra; les os des animaux sacrifiés. D'autres objets seront certainement présents, car le contexte n'a pas été entièrement fouillé.

Fig. 18 - Secteur Ouest : un choix des tessons de céramique grecque d'importation (avec quelques rares tessons indigènes) déposés entre la limite nord du sol US 640 et le creusement dans le sol géologique.

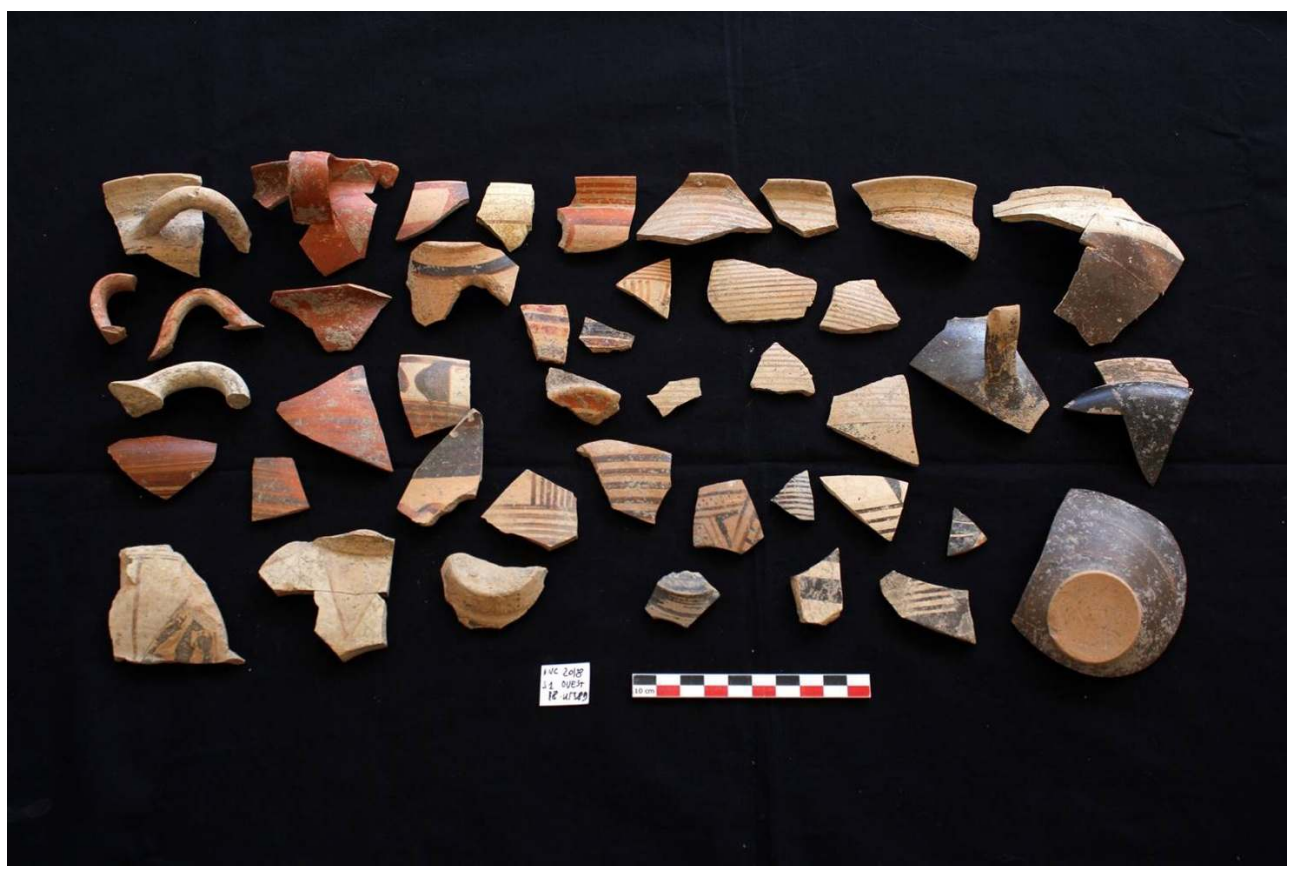

Cl. M. Denti.

Fig. 19 - Secteur Ouest : détail de la déposition au nord du sol US 640, avec l'amphore corinthienne, en cours de fouille.

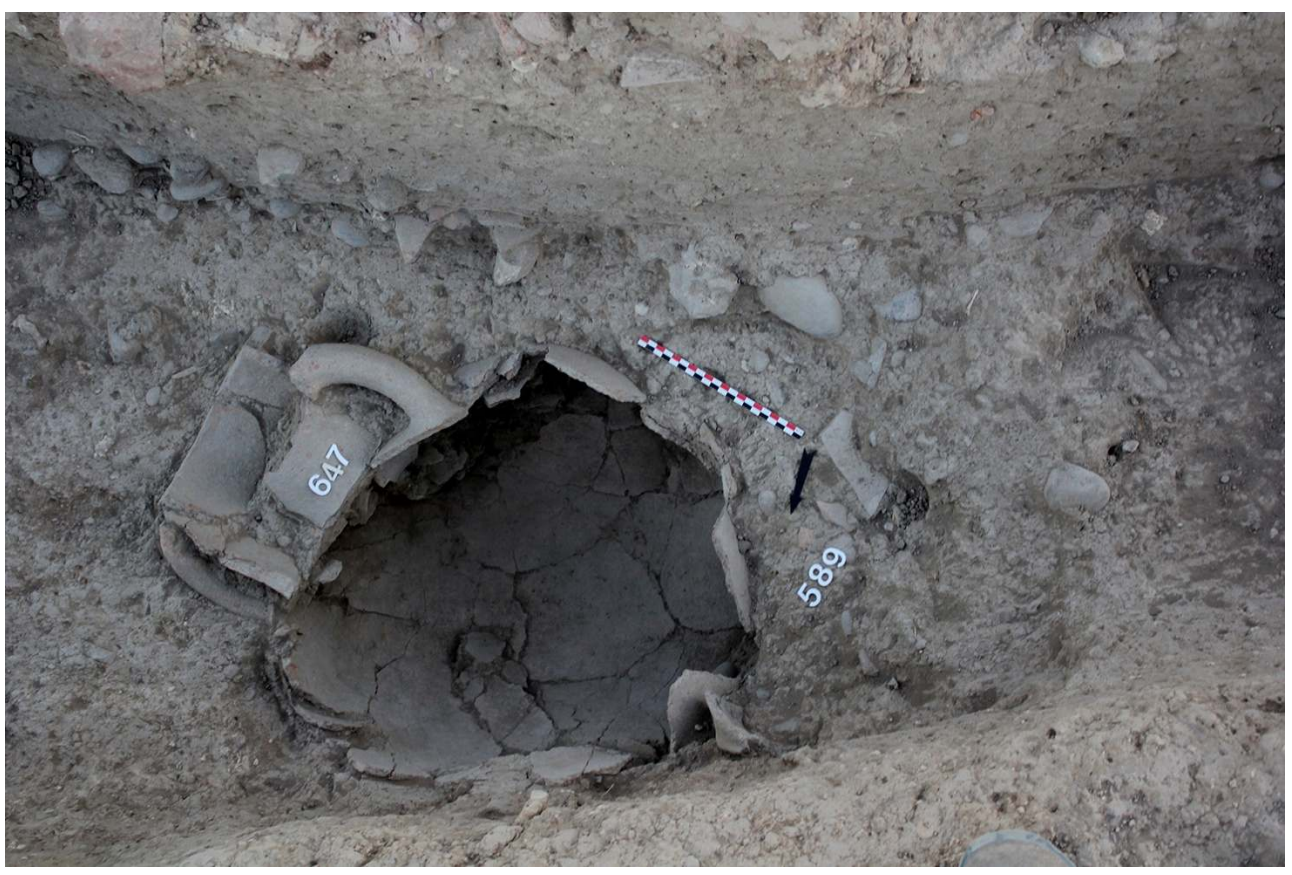

Cl. M. Denti. 
Fig. 20 - Secteur Ouest : col d'une œnochoé PC d'importation, de la déposition au nord du sol US 640 .

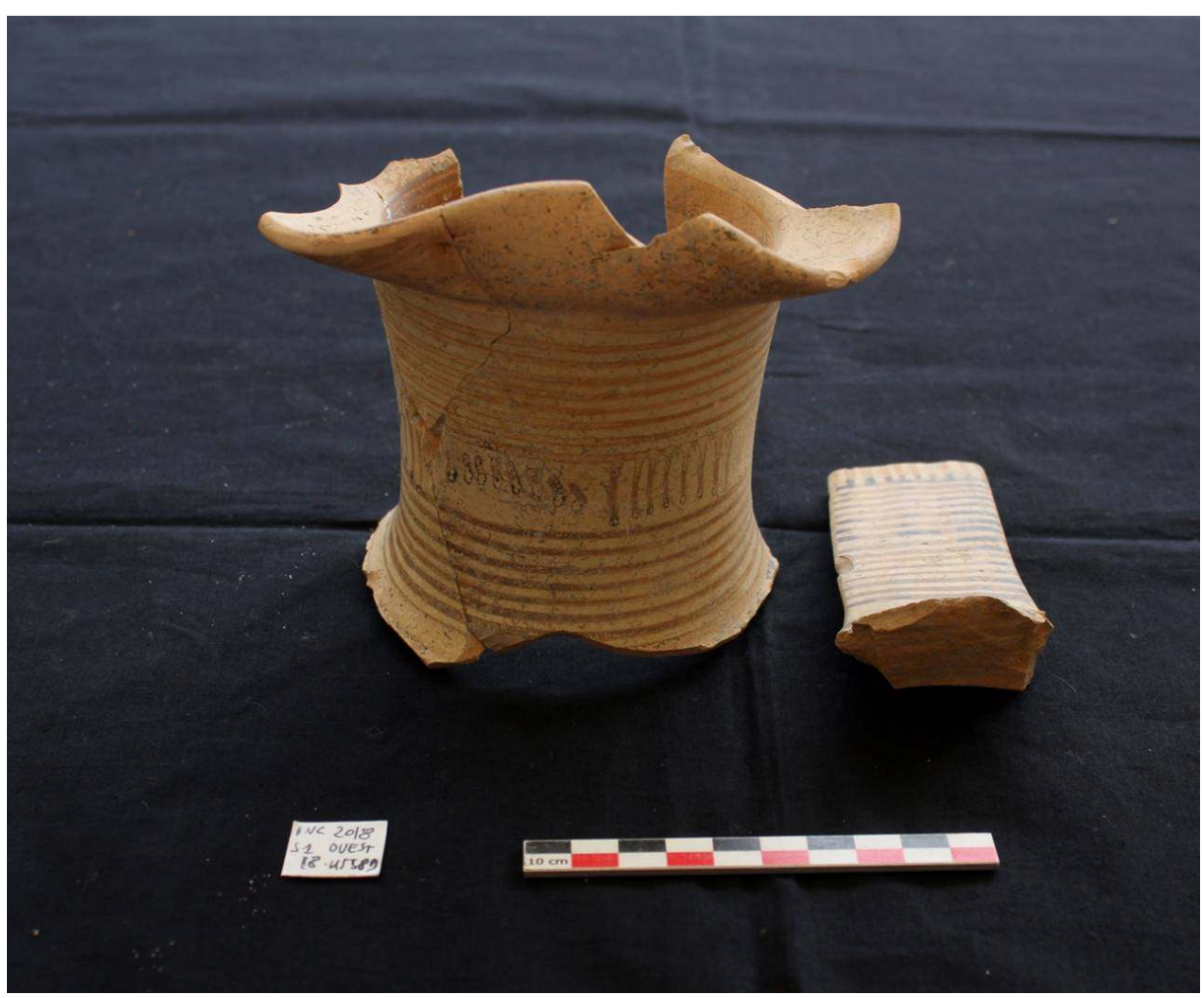

Cl. M. Denti. 
Fig. 21 - Secteur Ouest : krateriskos PC d'importation, de la déposition au nord du sol US 640.

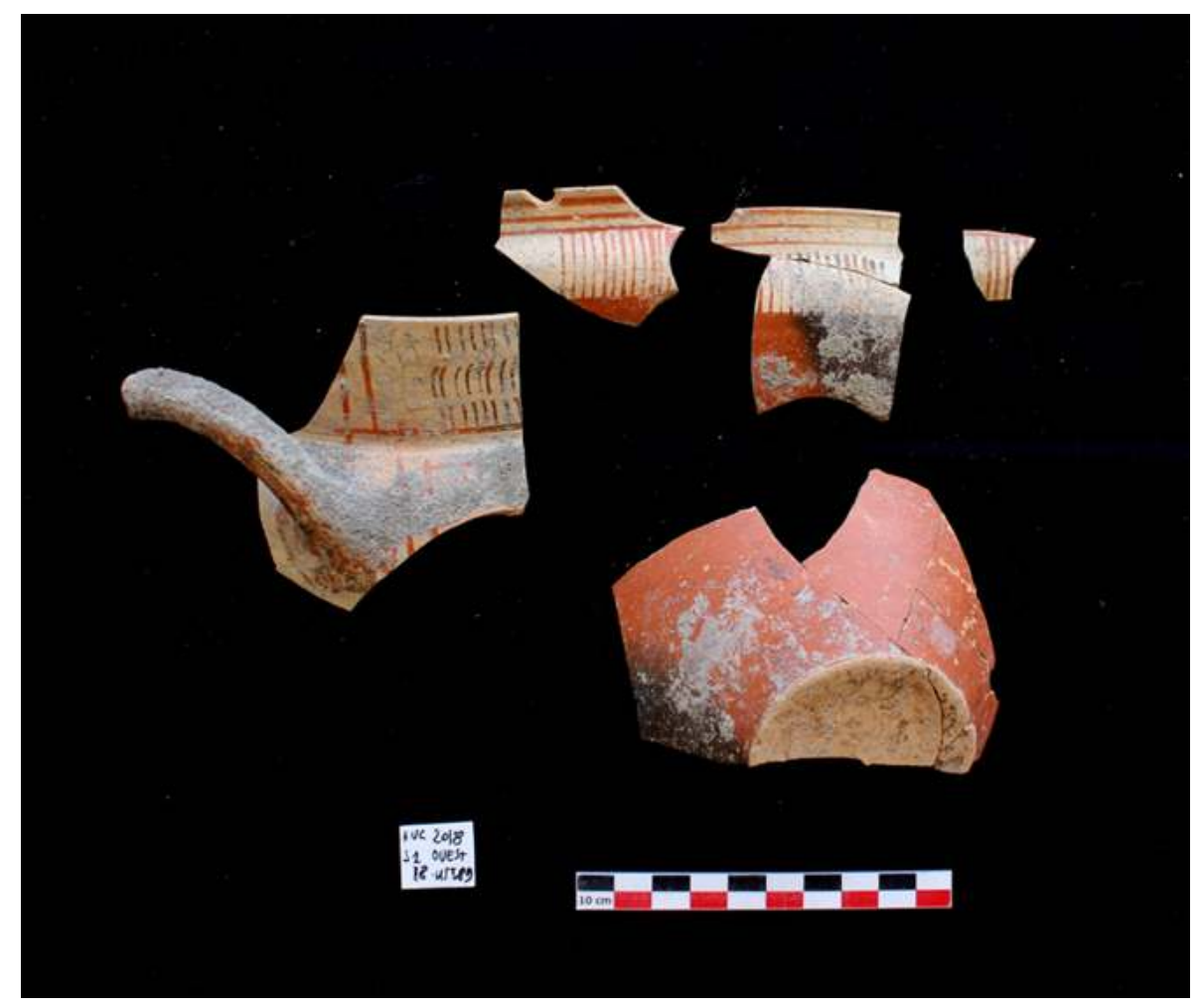

Cl. M. Denti.

Cette déposition pourrait correspondre à une pratique rituelle d'expiation, réalisée au moment de l'oblitération de l'édifice et caractérisée par un choix sélectif d'objets de prestige, importés de Grèce et - fait assez significatif - principalement de Corinthe. La présence de fragments appartenant aux mêmes vases, retrouvés à l'intérieur de ce contexte et, en même temps, dans la couche de recouvrement de la structure, semble suggérer une contemporanéité des deux opérations. Il semble ardu, pour le moment, d'en proposer une chronologie certaine. En effet, le matériel PC d'importation de chronologie assez haute (LPC ou MPC) se mélange avec des tessons LPC et à des fragments qui semblent appartenir à de la matt painted bichrome (fig. 18). En l'état actuel de nos connaissances, une date autour de la moitié du VII ${ }^{\mathrm{e}}$ siècle semble la plus probable.

Enfin, la coloration cultuelle de ce bâtiment a été ultérieurement corroborée par une découverte des plus remarquables mais, désormais, attendue. Une petite tête en bronze (présence possible de plomb dans l'alliage) a été déposée sur le sol (fig. 22), juste au milieu d'un ensemble de pierres disposées en forme de $\Pi$, qui semble l'encercler au nord (US 607, fig. 15, 17). Travaillée sur les deux côtés (à l'arrière, la chevelure est précisément décrite en relief), elle mesure $2 \times 2 \mathrm{~cm}$, avec une épaisseur d'1 $\mathrm{mm}$, et présente un probable dispositif d'attelage sur la partie supérieure (un anneau ?) (fig. 23). Réalisée en style dédalique, elle pourrait dater de la moitié du VII ${ }^{\mathrm{e}}$ siècle. 
Fig. 22 - Secteur Ouest : la petite tête dédalique en bronze, au moment de la découverte, posée sur le sol US 640 .

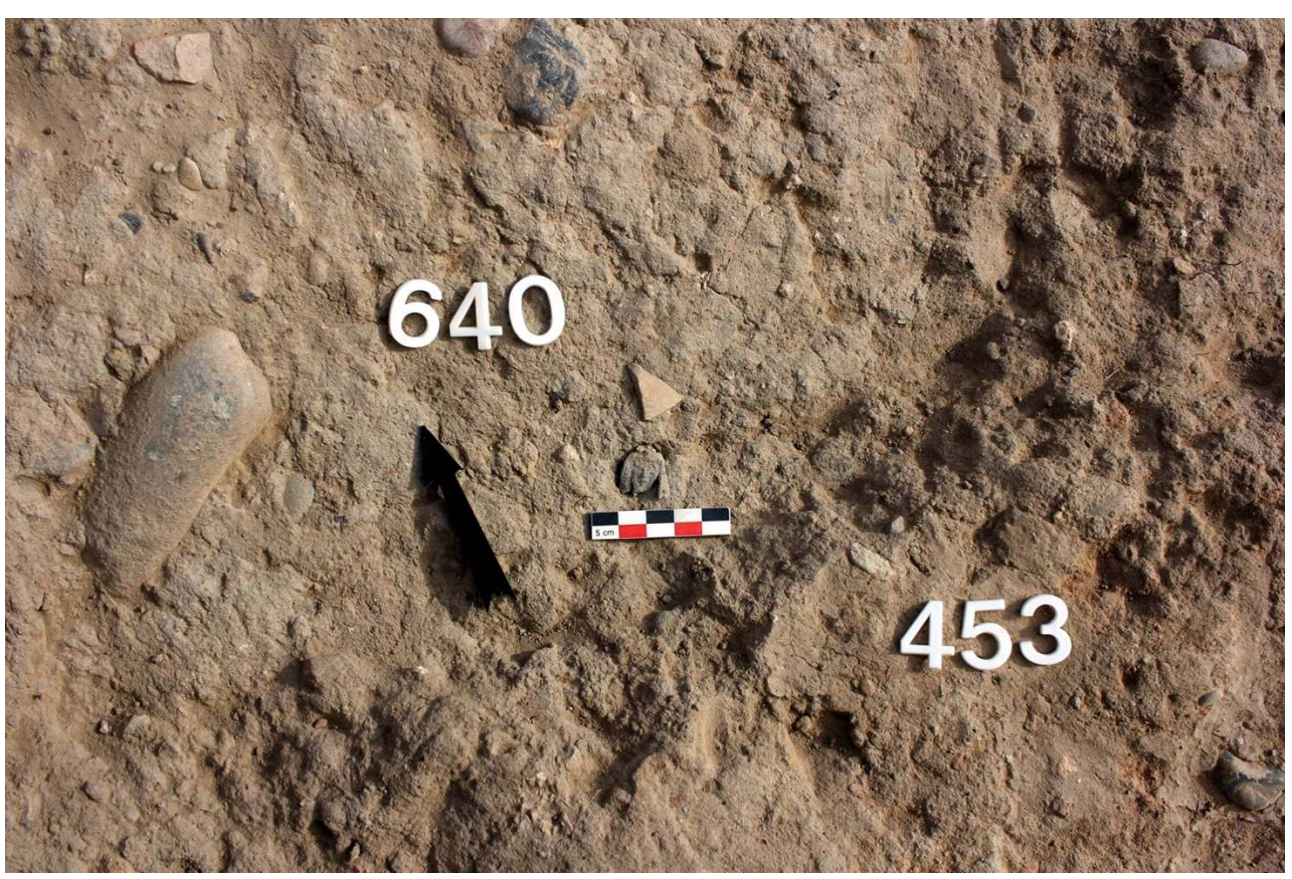

Cl. M. Denti.

Fig. 23 - Secteur Ouest : la petite tête dédalique en bronze, après restauration.

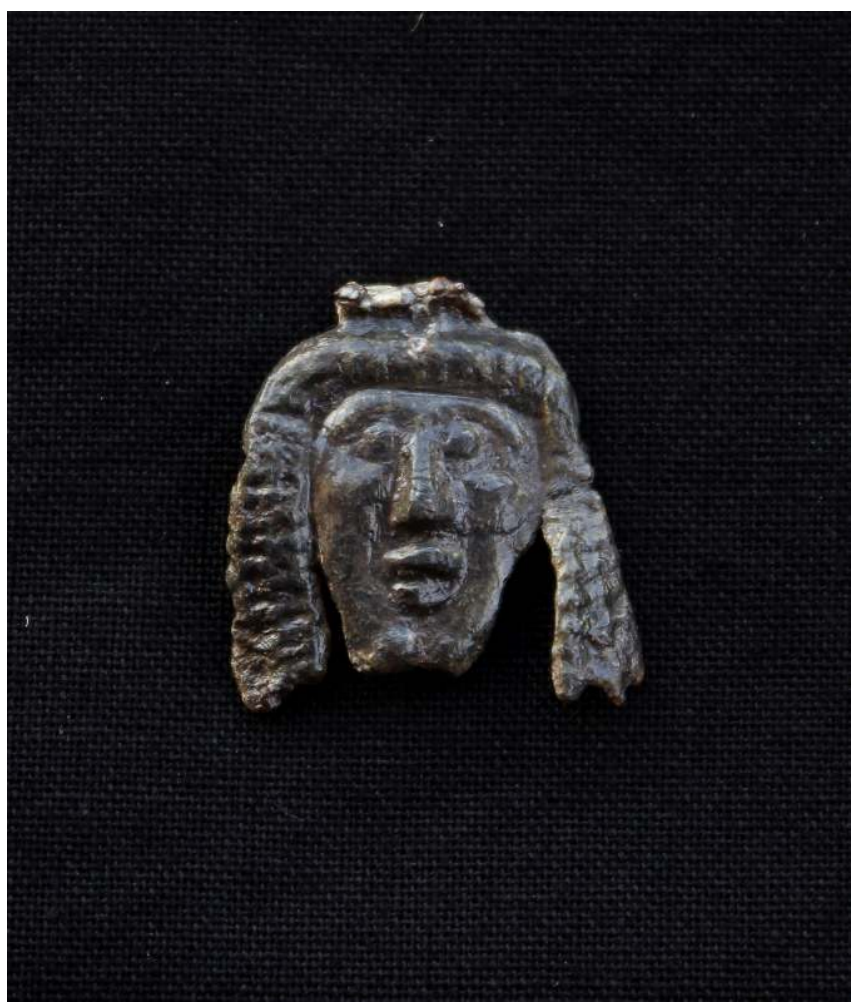

Cl. M. Denti. 
Cette découverte est très importante, non seulement en raison de la valeur historicoculturelle et historico-artistique de l'objet en soi (il s'agit de l'un des plus anciens documents de plastique « orientalisant » de la Grèce d'Occident) mais aussi et surtout, car cet objet tête a été retrouvé in situ, posé sur un sol appartenant à un bâtiment monumental qui, grâce aussi aux autres données contextuelles que nous venons de décrire, se qualifie comme un édifice à vocation religieuse.

\section{BIBLIOGRAPHIE}

Antonaccio 1995 = C.M. Antonaccio an archaeology of ancestors. Tomb cult and hero cult in early Greece, Lanham, 1995.

Bérard 1970 = C. Bérard, L'hérôon à la porte de l'Ouest, Berne, 1970 (Eretria, 3).

Brosse $1989=$ J. Brosse, Mythologie des arbres, Paris, 1989.

Denti 2013 = M. Denti, Incoronata. Les résultats de la dixième campagne de fouille (2012). Ruptures et continuités dans l'occupation du site entre VIII et ${ }^{e} \mathrm{VII}^{e}$ siècles avant J.-C., dans Chronique des activités archéologiques de l'École française de Rome [en ligne], 2013.

Denti 2015 = M. Denti, Incoronata. La douzième campagne de fouille (2014). Des nouvelles clés pour la compréhension de la nature du site, dans Chronique des activités archéologiques de l'École française de Rome [en ligne], 2015, https://cefr.revues.org/1327.

Denti 2017 = M. Denti, Topographie et fonction des sols, des fosses, des structures bâties : les résultats des campagnes de fouille de 2015 et 2016, dans Chronique des activités archéologiques de l'École française de Rome [en ligne], 2017.

Denti 2018 = M. Denti, Des pratiques rituelles perpétuées sur deux siècles d'occupation à Incoronata. La campagne de 2017, dans Chronique des activités archéologiques de l'École française de Rome [en ligne], 2018.

Denti sous presse $=$ M. Denti, Ritual pebbles. Pebbles between the living and the dead in the Mediterranean Iron Age, dans Festschrift G. Tsetskhladze, sous presse.

Denti - Lanos 2007 = M. Denti, Ph. Lanos, Rouges, non rougies. Les briques de l'Incoronata et le problème de l'interprétation des dépôts de céramique, dans MEFRA, 119-2, 2007, p. 445-481.

Fiorentino - Primavera 2014 = G. Fiorentino, M. Primavera, Acorn gatherers: fruit storage and processing in South-East Italy during the Bronze Age, dans Origini. Preistoria e Protostoria delle civiltà antiche, 35, 2013, p. 211-227.

Kourou $2015=$ N. Kourou, Early iron age mortuary contexts in the Cyclades. Pots, function and symbolism, in V. Vlachou (dir.), Pots, workshop and early iron age society: function and role of ceramics in early Greece. Proceeding of the International Symposium held at the Université libre de Bruxelles, 14-16 November 2013, Bruxelles, 2015, p. 83-105.

Lewthwaite 1982 = J.G. Lewthwaite, Acorns for Ancestors: the prehistoric exploitation of the woodland in the west Mediterranean, dans M. Bell, S. Limbray (dir.), Archaeological aspects of woodland ecology. 
Symposia of Association for Environmental Archaeology 2, Oxford, 1982 (BAR International series, 146), p. 217-230.

Margaritis 2017 = E. Margaritis, Domestic and ritual use of plants in early Iron Age Greece, dans A. Mazarakis Ainian, A. Alexandridou, X. Charalambidou (dir.), Regional stories towards a new perception of the early Greek world. Act of an International Symposium in honour of Professor Ian Bouzek, Volos 18-21 June 2015, Volos, 2017, p. 681-691.

\section{NOTES}

1. Toutes les dates sont avant J.-Chr.

2. Denti 2015, fig. 3-7.

3. Voir, pour la contextualisation et la signification de cette procédure, Denti sous presse.

4. Denti 2013, fig. 5-10.

5. Denti 2018, notamment fig. 4.

6. Bérard 1970 ; Antonaccio 1995.

7. Denti 2018, fig. 5.

8. Sur la valeur « héroïque » des tombes vides, voir dernièrement Kourou 2015.

9. Bérard 1970.

10. Denti 2013, fig. 11.

11. Denti 2017, fig. 4,6 .

12. Fiorentino - Primavera 2014.

13. Lewthwaite 1982 ; Brosse 1989, p. 71-108.

14. Margaritis 2017.

15. Denti 2018, fig. 7, 9-12.

16. Ibid.

17. Denti sous presse, avec des nombreuses comparaisons.

18. Denti 2018, fig. 15, 16.

19. Denti - Lanos 2007.

\section{INDEX}

Index géographique : Incoronata

Mots-clés : Âge du Fer, Italie du Sud, archéologie du rituel, cultes chtoniens, balanophagie institutions Laboratoire LAHM, UMR 6566 CReAAH, Université Rennes 2

\section{AUTEUR}

\section{MARIO DENTI}

Université Rennes 2, Laboratoire LAHM, UMR 6566 CReAAH - mario.denti@wanadoo.fr 